\title{
Almost sure behavior for increments of U-statistics
}

\author{
Dissertation \\ zur Erlangung des Doktorgrades \\ der Mathematisch-Naturwissenschaftlichen Fakultäten \\ der Georg-August-Universität zu Göttingen vorgelegt von
}

Mohammed Abujarad

aus

Gaza, Palestine

Göttingen, 2006 
D7

Referent: Prof. Dr. Manfred Denker

Korreferentin: Prof. Dr. Susanne Koch

Tag der mündlichen Prüfung: 18.01.2007 
Contents 


\section{Chapter 1}

\section{Introduction}

This work is concerned with a new type of almost sure behavior which was introduced by Erdös and Rényi in 1970 and which is called Erdös-Rényi law or new law of large numbers since then. They found that the maxima of partial sums of independent and identically distributed random variables summed over blocks of length $r_{n}$ converge almost surely after appropriate norming to some non-zero value. This value strongly depends on the growth rate of $r_{n}$ and is determined by the Laplace transform of the distribution. Moreover sometimes this value and the distribution of $X_{1}$ determined each other, provided that the growth rate of $r_{n}$ has order of logarithm.

A natural question is to generalize the result to more general averages. This is the main goal of this thesis.

To be more precise, suppose that $X, X_{1}, X_{2}, \ldots$ is a sequence of independent and identically distributed (i.i.d.) random variables defined on a probability space $(E, \mathcal{F}, P)$. To avoid trivialities, assume in addition that the $X_{i}^{\prime} s$ are nondegenerate, i.e. $P\left(X_{i}=x\right)<1$ for all $x$, with distribution function $F(x)=P(X<x)$. Let $S_{n}:=\sum_{i=1}^{n} X_{i}$ denote a partial sum of $X_{i}^{\prime} s$, with the convention that $S_{0}=0$. We are concerned with the almost sure (a.s.) limiting behavior of

$$
\begin{gathered}
T_{n}\left(r_{n}\right):=\left(S_{n+r_{n}}-S_{n}\right), \\
D_{n}\left(r_{n}\right):=\max _{0 \leq k \leq n-r_{n}}\left(S_{k+r_{n}}-S_{k}\right), \\
W_{n}\left(r_{n}\right):=\max _{0 \leq k \leq n-r_{n}} \max _{1 \leq j \leq r_{n}}\left(S_{k+j}-S_{k}\right),
\end{gathered}
$$




$$
R_{n}\left(r_{n}\right):=S_{n}-S_{n-r_{n}}, \text { and } \tilde{R}_{n}\left(r_{n}\right):=\max _{0 \leq k \leq r_{n}}\left(S_{n+k}-S_{n}\right)
$$

where $r_{n}$ is any nondecreasing sequence of natural numbers taking values between 1 and $n$. The exact form of $r_{n}$ will be specified later. The classical problems are as follows:

Problem (A): Under which conditions on the moments of $X_{i}$ and the growth rate of $r_{n}$, does there exists a norming sequence of constants $q_{n}$ such that with probability one

$$
\lim _{n \rightarrow \infty} \frac{D_{n}\left(r_{n}\right)}{q_{n}} \rightarrow \alpha \quad \text { or } \quad \limsup _{n \rightarrow \infty} \frac{D_{n}\left(r_{n}\right)}{q_{n}}=\alpha
$$

where $\alpha$ is a positive constant?

Furthermore, under the same conditions on the moment of $X_{i}$ and $r_{n}$, does the formula (??) still holds true, when we replace $D_{n}\left(r_{n}\right)$ by $W_{n}\left(r_{n}\right)$ or $T_{n}\left(r_{n}\right)$ or $R_{n}\left(r_{n}\right)$ ? Obviously $W_{n}\left(r_{n}\right) \geq D_{n}\left(r_{n}\right) \geq R_{n}\left(r_{n}\right)$ and $W_{n}\left(r_{n}\right) \geq \tilde{R}_{n}\left(r_{n}\right) \geq T_{n}\left(r_{n}\right)$.

Results of type (??) are important in many cases. For instance, in many practical situations, such as roulette or manufacturing, when observations are taken sequentially and each observation classified as success or failure. For these cases, it is possible to use (??) to test the hypothesis that the success probability is $p$. On the other hand (??) can be used to recognize pattern of unusually long runs of successes or failure, (see Arratia and Waterman [?]). Furthermore, such results and methods can be also used to study the asymptotic behavior of several statistics based on increments. For example, statistics of type $D_{n}\left(r_{n}\right)$ are applicable in actuarial and financial analysis which require estimates of possible losses or gains over time subinterval, (see, Bingswanger and Embrechts [?]).

These results are also used in investigating the asymptotic of increments of renewal processes, compound sum processes and other processes associated with sums $S_{n}$, (see, Steinebach [?], and Deheuvels and Steinebach 1989).

Moreover, such results are interesting from a probabilistic as well as statistical point of view since, on one hand, asymptotic properties of increments of certain stochastic processes can be characterized with probability one and, on the other hand, such properties even determine the whole underlying distribution in several cases, which is of definite statistical interest, (see e.g., [?], [?]).

Note that if $r_{n}$ grows as fast as $n$, then $D_{n}(n)=R_{n}(n)=S_{n}, W_{n}(n)=\max _{1 \leq k \leq n} S_{k}$, and if $r_{n}$ does not grow at all, say $r_{n}=1$, then $D_{n}(n)=W_{n}(n)=\max _{1 \leq k \leq n} X_{k}$. Hence, the theory of sums of random variables as well as the extreme value theory are parts of the general theory of statistics (??) - (??).

The a.s. asymptotic behavior of $D_{n}\left(r_{n}\right), W_{n}\left(r_{n}\right)$ and functionals of this type crucially depend on the growth rate of $r_{n}$. In the sequel we will distinguish between two kinds of increments: 
- Short increments, i.e., we let $r_{n}$ in the definition of the statistics defined by (??) (??) be any nondecreasing sequence of natural numbers such that

$$
r_{n}=O(\log n)
$$

holds. We will see in the next chapters that, the norming sequence $q_{n}$ in the theorems for short increments depends on the full distribution of $X$, and it sometimes characterizes this distribution. This observation has been made by Erdös and Rényi [?]. The case of short increments has been considered in a series of papers after the fundamental work of Erdös and Rényi. Various strong and weak limit theorems for short increments have been obtained by [?], [?, ?, ?], [?], [?, ?], [?], [?, ?], and [?]. The behavior of short increments has been studied earlier in 1964 by Shepp [?], where he introduced the statistic $T_{n}$. In fact D. J. Newman initiated the work related to the statistic $T_{n}$, where he consider the following situation:

Let $\left\{X_{i}: i \geq 1\right\}$ be independent and identically distributed Bernoulli trials with success probability $p \in(0,1)$. Let $N_{n}$ be the number of consecutive successes beginning at trial $n+1$ :

$$
\left\{\omega: N_{n}=j\right\}=\left\{\omega: X_{n+1}=1, X_{n+2}=1, \ldots, X_{n+j}=1, X_{n+j+1}=0\right\} .
$$

D. J. Newman proved that (see, e.g., [?] p. 61),

$$
\limsup _{n \rightarrow \infty} \frac{N_{n}}{\log _{1 / p} n}=1 \quad \text { a.s. }
$$

The results for short increments are called Erdös-Rényi and Shepp laws.

- Large increments, i.e. the sequence $r_{n}$ grow faster than $\log n$. It satisfies

$$
\frac{r_{n}}{\log n} \rightarrow \infty
$$

The norming sequence of theorems of large increments is relatively universal and it coincides with that for the Gaussian distribution. In 1979 Csörgö and Révéz [?] obtained the first result of this type by an application of the Komlós-Major-Tusnády strong approximation and results on asymptotics of large increments of a Wiener process (see, [?]). Frolov [?] has generalized these results to the case of one-sided moment assumption. These results are called the Csörgö-Révéz law. Thus, the asymptotic behavior of statistics (??) - (??) varies from strong noninvariance, like in Erdös-Rényi and Shepp laws to strong invariance, as in Csörgö-Révéz law.

An important role in modern probability theory and mathematical statistics is played by von Mises functionals and U-statistics, introduced by von Mises [?] and by Hoeffding 
respectively. The origin of U-Statistic can be traced back to the fundamental work of Hoeffding in 1948, as a generalization of the sample mean. Hoeffding proved the central limit theorem for U-statistics. His work was influenced by Halmos [?] and was also closely related to von Mises. Of particular interest is the fact that many estimate and statistics for tests of hypotheses can be represented as von Mises functionals and U-statistics. The use of U-statistics is an effective way to obtain an unbiased estimator. In nonparametric problems, U-statistics are often uniformly minimum variance unbiased estimators (UMVUE), where as in parametric problems, U-statistics can be used as initial estimators to derive more efficient estimators. In addition to numerous articles, there are several monographs devoted to properties of U-statistics (see, Denker [?], Lee [?], Borovskikh [?] or Serfling [?] chapter $5-6)$.

The limit theorems for increments of sums of i.i.d. random variables as introduced above are well understood in the sense that there are necessary and sufficient analytic conditions under which the Problem (A) is completely solved. As U-statistics generalize the concept of usual mean of a sequence of independent and identically distributed random variables, they still need to be developed in that sense. Towards that goal we extend Erdös-Rényi and Shepp laws as well as Csörgö-Révéz law for U-statistics in our thesis. Precisely we would like to do the following:

For a nondecreasing sequence of natural numbers $r_{n}$ so that $m \leq r_{n} \leq n$, we introduce two different types of statistics based on increments of U-statistics of degree $m$,

$$
\hat{D}_{n}\left(r_{n}\right)=\max _{0 \leq k \leq n-r_{n}}\left\{\left(k+r_{n}\right)\left(U_{k+r_{n}}(h)-\vartheta\right)-k\left(U_{k}(h)-\vartheta\right)\right\},
$$

and

$$
D_{n}\left(r_{n}\right):=\max _{0 \leq k \leq n-r_{n}} U_{1+k}^{k+r_{n}}:=\max _{0 \leq k \leq n-r_{n}} \sum_{1+k \leq i_{1}<\ldots<i_{m} \leq k+r_{n}} h\left(X_{i_{1}}, \ldots, X_{i_{m}}\right) .
$$

- Observe that if $m=1$ and $\vartheta:=E h\left(X_{1}\right)=0$, then

$$
D_{n}\left(r_{n}\right)=\hat{D}_{n}\left(r_{n}\right)=\max _{0 \leq k \leq n-r_{n}}\left\{\sum_{j=1}^{n+r_{n}} h\left(X_{j}\right)-\sum_{j=1}^{k} h\left(X_{j}\right)\right\} .
$$

Thus the statistics $D_{n}\left(r_{n}\right)$ and $\hat{D}_{n}\left(r_{n}\right)$ coincide with the statistic $D_{n}\left(r_{n}\right)$ introduced by Erdös and Rényi. Therefore, in the sequel we will consider the case that $m \geq 2$. 
- Observe that if $r_{n}$ take the extreme values, then

$$
D_{n}(n)=\left(\begin{array}{c}
n \\
m
\end{array}\right) U_{n}(h), \quad D_{n}(m)=\max _{0 \leq k \leq n-m} h\left(X_{k+1}, \ldots, X_{k+m}\right)
$$

and

$$
\begin{gathered}
\hat{D}_{n}(m)=\max _{0 \leq k \leq n-m}\left\{(k+1)\left(U_{k+1}(h)-\vartheta\right)-k\left(U_{k}(h)-\vartheta\right)\right\}, \\
\hat{D}_{n}(n)=n\left(U_{n}(h)-\vartheta\right) .
\end{gathered}
$$

- Let $m=2$ and $h$ be given by $h(x, y)=(x+y) / 2$ with $E h=\vartheta=0$, then

$$
\hat{D}_{n}\left(r_{n}\right):=\max _{0 \leq k \leq n-r_{n}}\left(S_{k+r_{n}}-S_{k}\right), \quad D_{n}\left(r_{n}\right):=\max _{0 \leq k \leq n-r_{n}}\left(S_{k+r_{n}}-S_{k}\right) /\left(r_{n}-1\right) .
$$

Hence, the theory of sums of U-statistics, the extreme value theory for $m$ dependent random variables as well as the statistics (??) given by Erdös and Rényi are parts of the general theory of statistics (??), (??). In fact we go further than statistics (??) and (??) in the sequel and call them functionals of type $\hat{D}_{n}\left(r_{n}\right)$ or $D_{n}\left(r_{n}\right)$.

After defining statistics (??) - (??), the main object in our thesis is to find a norming sequence $c_{n}$ and sufficient conditions on the moments of $h$, which stabilize the statistics (??) - (??) non trivially with probability one. Formally, we want to find norming sequence $c_{n}$ and sufficient conditions on $h$ implies that

$$
\lim _{n \rightarrow \infty} \frac{\hat{D}_{n}\left(r_{n}\right)}{c_{n}} \rightarrow \alpha
$$

and

$$
\lim _{n \rightarrow \infty} \frac{D_{n}\left(r_{n}\right)}{c_{n}} \rightarrow \alpha
$$

where $\alpha$ is a positive number and $\alpha, c_{n}$ in (??), (??) are not necessary the same. We replace lim by limsup, if the lim in (??) and (??) does not exist. In fact, in the case $m \geq 2$ this problem become significantly more complicated, and no general results in this area until recently.

The organization of this thesis is the following:

In Chapter ?? we briefly review some basic facts of U-statistics and fix our notations. In particular, we remark the martingale property of $U_{1+k}^{k+r_{n}}$, which will be essential for proving the upper limit of Csörgö-Révéz law for U-statistics in Chapter ??.

In Chapter ??, under suitable conditions on $h$, we prove statement (??) for $\hat{D}_{n}\left(r_{n}\right)$ and for other functionals of type $\hat{D}_{n}\left(r_{n}\right)$ defined in this chapter (see, Corollaries ?? and 
??). Our analysis is based on the upper limit for the statistics $\hat{T}_{n}\left(r_{n}\right)$ which is stated in Proposition ??. Taking $h(x)=x$ we note that $\hat{D}_{n}\left(r_{n}\right)$ reduces to $D_{n}\left(r_{n}\right)$ defined by (??). Hence we recover the result by Frolov. For the sake of completeness we have stated Frolov's result in Theorem ??. It is well known that Frolov's theorem implies the ErdösRényi and Shepp laws as well as Csörgö-Révéz law, and we show in section ?? that these property also hold for kernels with dimension greater than or equal 2. In Subsection ?? we give an application of Corollary ?? including Erdös-Rényi and Shepp type laws, the Csörgö-Révéz law and the law of iterated logarithm for nondegenerate U-statistics. Mason's theorem also holds partly for these statistics. Thus we can get Erdös-Rényi and Shepp type laws, Csörgö-Révéz law and the Mason theorem as a by-product. For the reader's convenience we state these known results in the first three sections.

In Chapter ?? we recall some well known results from large deviations theory for Ustatistics and derived from them some corollaries as well as we proved some axillary lemmas, which will play an important role in describing the a.s. asymptotic behavior of the statistics $D_{n}\left(r_{n}\right)$ and functionals of this type in Chapter ??.

In Chapter ?? we prove another type of Erdös-Rényi law for arbitrary U-statistics with kernel of degree $m=2$ (Theorem ??). Thus we solve statement (??) partly, in the sense that, the a.s. asymptotic behavior of the statistics $D_{n}\left(r_{n}\right)$ defined by (??) is described only for sequences $r_{n}$ such that $r_{n}=[c \log n]$. Hence, by definition of $D_{n}\left(r_{n}\right)$ in (??), Theorem ?? generalize Theorem ?? given by Erdös-Rényi 1970. Furthermore we extend Theorem ?? to describe the a.s. asymptotic behavior of the statistics $D_{n}\left(r_{n}\right)$ defined by (??) considering increments of length $(\log n)^{p}$ for $p>1$. Thus we solve statement (??) for $r_{n}=(\log n)^{p}$ for $p>1$. This is done in Theorem ?? and Theorem ??. These theorems hold only for nondegenerate U-statistics with kernel of degree $m \geq 2$ and generalize Theorem ?? given by Book [?]. Hence Theorem ?? together with Theorem ?? solve statement (??) for $r_{n}=(\log n)^{p}$ with $p \geq 1$ completely.

However we are only able to give the lower limit of Csörgö-Révéz law for nondegenerate U-statistics, and the upper limit of Csörgö-Révéz law for arbitrary U-statistics. This is done in Lemma ?? and Lemma ?? respectively. These lemmas generalizes Theorem ?? given by Frolov [?] partly. That means the statement (??) is solved for $(\log n) \leq r_{n} \leq n$ partly.

Finally to solve statement (??) completely, we describe the a.s. asymptotic behavior of the statistics $D_{n}\left(r_{n}\right)$ defined by (??) considering increments $r_{n}$ such that $\frac{r_{n}}{\log n} \rightarrow 0$ partly, i.e., we give only the upper limit. This is done for arbitrary U-statistics with kernel of degree $m$ and given by Lemma ??. Thus we generalizes Theorem ?? given by Mason [?] partly. We also partly extend Theorem Theorem ?? for arbitrary U-statistics with kernel of degree $m \geq 2$ and given by Lemma??. 
Chapter ?? contains a short summary and discussion of open problems to be considered in further investigations. We give also a few references, which may be helpful for further extension of our results.

\section{Acknowledgements}

I am most grateful to my supervisor, Prof. Dr. Manfred Denker, for proposing the topic of my dissertation, his excellent supervision and many helpful suggestions during my study. I would also like to thank him for his patience, encouragement and supporting me financially. I am also grateful to my coadvisor Prof. Dr. Susanne Koch for her encouraging and accepting to be coadvisor of this thesis. My special thanks go to Prof. Dr. Edgar Brunner and Prof. Dr. Axel Munk for their support.

I am grateful to all the people from the Institute for Mathematical Stochastics for providing me with a nice working environment and helpful discussion. Particular thanks go to Achim Wübker, Sachar Kablutschko, Dr. Alexei Min, Dr. Hajo Holzmann, Dr. Leif Boysen, and all my other colleagues and friends.

My heartiest thanks go to my mother, father, Wéam, Alshimaa, and my wife Rania for supporting me with patience, love and understanding. I gratefully acknowledge the financial support and encouragement from the KAAD. The work would not be completed without their active involvement in the project. My special thanks go to Gabrielle Beitzel and Hans Landsberg. 


\section{Chapter 2}

\section{Basic General Facts}

In this chapter, we give a brief overview on some properties of U-statistics needed in the sequel. We base the terminology of the general theory of U-statistics on Denker [?], Lee [?] or Borovskikh [?]. See also Serfling [?] chapter 5-6. Hoeffding decomposition and U-statistics with its Martingale property are represented in the first section. In the second section of this chapter notations from large deviation theory for sums of i.i.d. random variables are introduced. We give a few examples corresponding to the notations.

\subsection{Basic Definition}

Following [?], we introduce the necessary notations. Suppose that $X, X_{1}, X_{2}, \ldots$ is a sequence of i.i.d. random variables defined on a probability space $(E, \mathcal{F}, P)$. For an arbitrary measurable space $(E, \mathcal{F})$ we denote by $\mathcal{P}$ the family of all probability measures on $\mathcal{F}$ and $\mathcal{P}_{0} \subset \mathcal{P}$. Based on this notation, the functional $\vartheta: \mathcal{P}_{0} \rightarrow \mathbb{R}$ is called regular or estimable with respect to $\mathcal{P}_{0}$, if there exists an integer $m \geq 1$ and a measurable map $h: E^{m} \rightarrow \mathbb{R}$ such that

$$
E h=\int h d P^{m}=\vartheta(P)
$$

for all $P \in \mathcal{P}_{0}$, where $P^{m}$ is the m-fold Cartesian product of $P$. Based on the regular function $\vartheta(P):=\vartheta$, we are able to give the following

Definition 2.1.1. Let $h: E^{m} \rightarrow \mathbb{R}$ be a measurable map and symmetric in its arguments. 
Then the map

$$
U_{n}(h)=\left(\begin{array}{c}
n \\
m
\end{array}\right)^{-1} \sum_{1 \leq i_{1}<\ldots<i_{m} \leq n} h\left(X_{i_{1}}, X_{i_{2}}, \ldots, X_{i_{m}}\right),
$$

is called a U-statistics with kernel $h$ of degree $m$. The map

$$
V_{n}(h)=n^{-m} \sum_{1 \leq i_{1}, \ldots, i_{m} \leq n} h\left(X_{i_{1}}, X_{i_{2}}, \ldots, X_{i_{m}}\right)
$$

is known as $V$-statistics or von Mises-statistics with kernel h of degree $m$.

Notice that in the above definition, we can always assume that $h$ is symmetric in its arguments. This follows from the fact that when $h$ defines an unbiased estimator of $\vartheta(P)$, then the function

$$
h^{\star}\left(x_{1}, x_{2}, \ldots, x_{m}\right)=\frac{1}{m !} \sum_{\tau \in P e r} h\left(x_{\tau(1)}, x_{\tau(2)}, \ldots, x_{\tau(m)}\right)
$$

is symmetric in its arguments and is also unbiased estimator of $\vartheta(P)$, where the sum extends over all permutations of $\{1, \ldots, m\}$. Since $h$ and $h^{\star}$ have the same expectation under $P$, one can replace $h$ by $h^{\star}$.

$U_{n}(h)$ can be regarded as the unbiased variant of $V_{n}(h)$. Well known examples of Ustatistics are the empirical $k$ th moment, Gini's mean differences, one-sample Wilcoxon statistic, etc.

\subsubsection{Hoeffding Decomposition and Representation of U-Statistics}

A U-statistics by construction is an average of dependent observations except in the trivial case where $m=1$. However, a U-statistic can be represented as average of dependent averages of i.i.d. random variables, i.e.

$$
U_{n}(h)-\vartheta=\frac{1}{n !} \sum_{P e r .} W\left(X_{i_{1}}, \ldots, X_{i_{n}}\right),
$$

where $\sum_{\text {Per. }}$ denotes summation over all permutations $\left(i_{1}, \ldots, i_{n}\right)$ of $(1, \ldots, n)$ and

$$
W\left(X_{1}, \ldots, X_{n}\right)=[n / m]^{-1} \sum_{p=1}^{[n / m]} h\left(X_{m p-m+1}, \ldots, X_{m p}\right) .
$$


The representation in formula (??) is called Hoeffding representation, due to Hoeffding (1963).

Definition 2.1.2. Suppose that $h: E^{m} \longrightarrow \mathbb{R}$ is an unbiased estimator of $\vartheta$, then we call $h$ degenerate kernel with respect to $P \in \mathcal{P}_{0}$ if

$$
\int h\left(x_{1}, x_{2}, \ldots, x_{m}\right) d P\left(x_{1}\right)=0, \quad \forall x_{2}, \ldots, x_{m}
$$

For a given kernel $h: E^{m} \rightarrow \mathbb{R}$ and a fixed probability measure $P \in \mathcal{P}_{0}$ satisfying the condition $\int\left|h\left(x_{1}, \ldots, x_{m}\right)\right| d P\left(x_{1}\right) \ldots d P\left(x_{m}\right)<\infty$, consider the functions

$$
\tilde{h}_{c}\left(x_{1}, \ldots, x_{c}\right):=E\left(h\left(X_{1}, \ldots, X_{m}\right) \mid X_{1}=x_{1}, \ldots, X_{c}=x_{c}\right)
$$

and

$$
h_{c}\left(x_{1}, \ldots, x_{c}\right):=\sum_{k=0}^{c}(-1)^{c-k} \sum_{K \subseteq\{1, \ldots, c\},|K|=k} \tilde{h}_{k}\left(\mathbf{x}_{K}\right)
$$

where $\mathbf{x}_{K}=\left(x_{i_{1}}, \ldots, x_{i_{k}}\right) \in E^{k}$ such that $\left\{i_{1}<\ldots<i_{k}\right\} \subseteq\{1, \ldots, c\}$ for $c=0,1, \ldots, m$. According to Definition ?? one can know easily that the functions $h_{c}\left(x_{1}, \ldots, x_{c}\right)$ are degenerate and symmetric. Furthermore, we will see later how the functions $\tilde{h}_{c}$ and $h_{c}$ play an important rule in establishing limit theorems for U-statistics.

Definition 2.1.3. Let the kernel $h\left(x_{1}, \ldots, x_{m}\right)$ satisfy $E h^{2}\left(x_{1}, \ldots, x_{m}\right)<\infty$. For $c=$ $1, \ldots, m$, define $\zeta_{c}^{2}:=\operatorname{Var}\left(\tilde{h}_{c}\left(x_{1}, \ldots, x_{c}\right)\right)$. Then, we say that the kernel $h$ or the $U$-statistics $U_{n}(h)$ possess a degeneracy of order $c$ if $\zeta_{j}=0$ a.s. for $j \leq c$ and $\zeta_{c+1}>0$.

In what follows, we will make use of the following. For a given i.i.d. random variables $X_{1}, X_{2}, \ldots$ with a probability distribution $F$, let $h(x, y)$ be symmetric, square integrable, i.e.

$$
\iint h^{2}(x, y) d F(x) d F(y)<\infty
$$

and degenerate, i.e. $\int h(x, y) d F(x)=0$ for all $y$. Let $L^{2}$ be the space of all square integrable functions with respect to $F \otimes F$. Then (see e.g. Serfling [?] p. 193), we see that the kernel $h$ induces a bounded linear operator $T_{h}: L^{2} \rightarrow L^{2}$ defined by $T_{h} f(x):=$ $E h\left(X_{1}, x\right) f\left(X_{1}\right)$, which has eigenvalues $\beta_{i}$ and the corresponding eigenfunctions $\psi_{i}(X)$ satisfying the properties:

$$
E \psi_{i}\left(X_{1}\right)=0, E \psi_{i}^{2}\left(X_{1}\right)=1, E \psi_{i}\left(X_{1}\right) \psi_{j}\left(X_{1}\right)=0
$$

for $i \neq j, i, j \geq 1$ and $E h\left(X_{1}, x\right) \psi_{i}\left(X_{1}\right)=\beta_{i} \psi_{i}(x)$. Moreover, for each $i, j$ with $i \neq j$ the 
expansion

$$
\lim _{n \rightarrow \infty} E\left(h\left(X_{i}, X_{j}\right)-\sum_{p=1}^{n} \beta_{p} \psi_{p}\left(X_{i}\right) \psi_{p}\left(X_{j}\right)\right)^{2}=0
$$

holds. We remark that the operator $T_{h}$ is Hilbert- Schmidt operator.

For the sake of completeness, we next introduce a theorem, derived by Hoeffding in 1961. Its importance lies on the fact that any kernel $h$ of degree $m$ can be written as a sum of degenerate kernels $h_{c}$ of degrees $c=0,1, \ldots, m$, where the terms $U_{n}\left(h_{c}\right)$ are uncorrelated with variances of decreasing order in $n$.

Theorem 2.1.1. (H-decomposition Theorem).

Let $h: E^{m} \longrightarrow \mathbb{R}$ be symmetric unbiased estimator of $\vartheta$. Then for any $P \in \mathcal{P}_{0}$ there exist degenerate symmetric kernels $h_{c}: E^{c} \longrightarrow \mathbb{R}$ of degree $c=0,1, \ldots, m$. Moreover $U_{n}(h)$ admits the representation

$$
U_{n}(h)-\vartheta=\sum_{c=1}^{m}\left(\begin{array}{l}
m \\
c
\end{array}\right) U_{n}\left(h_{c}\right),
$$

where $U_{n}\left(h_{c}\right)$ is $U$-statistics based on a degenerate kernel of degree c.

\subsubsection{Martingale Property of U-Statistics}

In this subsection we will mention several lemmas of martingale property of U-statistics needed in the next chapter. In 1961 Hoeffding derived the following lemma, and in fact, used it to establish the strong law of large numbers for U-statistics.

Lemma 2.1.1. Let $h$ be a degenerate kernel of degree $m$, based on the sequence $\left\{X_{n}: n \geq\right.$ $m\}$ of i.i.d. random variables. Then $\left\{\left(\begin{array}{c}n \\ m\end{array}\right) U_{n}(h): n \geq m\right\}$ is a martingale with respect to the filtration $\mathcal{F}_{n}:=\sigma\left(X_{1}, X_{2}, \ldots, X_{n}\right)$.

Berk [?] proved that $U_{n}(h)$ can also be viewed as a reverse martingale with respect to the filtration $\mathcal{F}_{n}=\sigma\left(\mathbf{X}_{(n)}, X_{n+1}, X_{n+2}, \ldots\right)$, where $\mathbf{X}_{(n)}:=\left(X_{(1)}, \ldots, X_{(n)}\right)$ is a vector of ordered statistic for $n$ random variables. He used this property together with a reverse martingale convergence theorem to give a new proof of SLLN for U-statistics.

Lemma 2.1.2. Let $\left\{U_{n}(h): n \geq m\right\}$ be a sequence of $U$-statistics and suppose that the condition $E\left|h\left(X_{1}, X_{2}, \ldots ., X_{m}\right)\right|<\infty$ holds. Then $\left\{U_{n}(h): n \geq m\right\}$ is a reverse martingale adapted to the $\sigma$-algebras $\mathcal{F}_{n}=\sigma\left(\mathbf{X}_{(n)}, X_{n+1}, X_{n+2}, \ldots\right)$. 
Remark 2.1.1. Let $r_{n}$ be a nondecreasing sequence of natural numbers such that $m \leq$ $r_{n} \leq n$. Then $\left\{U_{1+k}^{k+r_{n}}: 0 \leq k \leq n-r_{n}\right\}$ is a reverse martingale adapted to the $\sigma$ - algebras $\mathcal{F}_{k}:=\sigma\left(U_{j+k}^{j+k+r_{n}}: j \geq 1\right)$, where $U_{1+k}^{k+r_{n}}$ defined by (??). The proof of this fact is similar to that given by Berk [?].

Lemma 2.1.3. Let $c_{n}, n \geq m$, be a nondecreasing sequence of positive numbers and suppose that $E h^{2}\left(x_{1}, \ldots, x_{m}\right)<\infty$. Then

$$
P\left\{\max _{n \leq p \leq N} c_{p}\left|U_{p}(h)\right| \geq \lambda\right\} \leq \lambda^{-2}\left(c_{n}^{2} \operatorname{Var}\left(U_{n}(h)\right)+\sum_{n=p+1}^{N}\left(c_{p}^{2}-c_{p-1}^{2}\right) \operatorname{Var}\left(U_{p}(h)\right)\right),
$$

for all $m \leq n \leq N$ and $\lambda>0$. If the series on the right hand side is convergent,then $\max _{n \leq p \leq N}$ and $N$ can be replaced by $\sup _{p \geq n}$ and $\infty$ respectively.

\subsection{Notations and Examples.}

Assumptions (A): Throughout this thesis we have the following assumptions and notations for our convenience:

- We suppose that $X, X_{1}, X_{2}, \ldots$ is a sequence of i.i.d. random variables defined on a probability space $(E, \mathcal{F}, P)$.

- When we mention that the sequence $X, X_{1}, X_{2}, \ldots$ satisfies the condition (H), we are referring to the following three conditions:

1. $0 \leq E(X)<\infty$,

2. $X$ is nondegenerate, i.e., for all $x, P\left(X_{1}=x\right)<1$,

3. $t_{0}:=\sup \left\{t: E\left(e^{t X}\right)<\infty\right\}>0$.

For such random variables a collection of basic functions and constants are introduced and their properties are investigated, which play an essential role in describing the asymptotic limiting behavior of the statistics (??) - (??). For $0<t \leq t_{0}$, define the following:

$$
\begin{gathered}
\varphi(t):=E\left(e^{t X}\right), \quad m(t):=\frac{\varphi^{\prime}(t)}{\varphi(t)}, \quad \sigma^{2}(t):=m^{\prime}(t) . \\
A:=\lim _{t \nearrow t_{0}} m(t), \quad f(t):=t m(t)-\log \varphi(t), \quad \frac{1}{c_{0}}=\lim _{t \nearrow t_{0}} f(t) .
\end{gathered}
$$




$$
\begin{array}{r}
\xi(\alpha):=\sup _{t \in \mathbb{R}}\{t \alpha-\log \varphi(t): \varphi(t)<\infty\}, \quad \omega:=\text { ess } \sup X \\
-\log \rho(\alpha):=\xi(\alpha), \quad \gamma(x):=\sup \{\alpha: \xi(\alpha) \leq x\} .
\end{array}
$$

The functions $m(t)$ and $\sigma^{2}(t)$ are well known from the theory of large deviations. They are mean and variance of the Cramér transformation of $X$ respectively. The function $\xi(x)$ is called Legendre transform of cumulant generating function $\log E e^{t X_{1}}$ or rate function. The computation of the function $\gamma(x)$ is difficult in general, and closed-form expression are only known for special distributions.

- $a_{n}$ will stand for a nondecreasing sequence of natural numbers such that $1 \leq a_{n} \leq n$. In addition to $a_{n}$ we assume that there exists a nondecreasing function of real numbers $a(x)$ such that the following three conditions holds

1. $a_{n} / a(n) \rightarrow 1$,

2. $1 \leq a(x) \leq x$, and

3. $x / a(x)$ is nondecreasing.

- $\beta_{n}$ will always be given by $\beta_{n}:=\log \left(n / a_{n}\right)+\log \log n$.

- $b_{n}$ will stand for a nondecreasing sequence of numbers given by

$$
b_{n}:=a_{n} \gamma\left(\frac{\beta_{n}}{a_{n}}\right)
$$

and its associated nondecreasing function

$$
b(x):=a(x) \gamma\left(\frac{\beta(x)}{a(x)}\right) \text { with } \beta(x):=\log (x / a(x))+\log \log x .
$$

- $[x]$ will stand for the integer part of $x$.

The following lemma characterizes the properties of the function defined by (??) - (??), and its proof can be found in Deheuvels et. al [?], Mason [?] and Petrov [?].

Lemma 2.2.1. For a given random variable $X$ satisfying condition (H), we have the following results:

1. $m(0)=E X, m(t)$ is strictly increasing on $\left[0, t_{0}\right)$ and continuously differentiable on $\left(0, t_{0}\right)$.

2. For any $t \in\left[0, t_{0}\right)$, we have $m(t) \in[E X, A)$, conversely for any $\alpha \in(E X, A)$, there exist a unique $\bar{t}:=t(\alpha) \in\left(0, t_{0}\right)$ such that $m(\bar{t})=\alpha$. 
3. For any $c \in\left(c_{0}, \infty\right)$, there exists a unique $\alpha \in(E X, A)$ such that $c=c(\alpha)$.

4. For any $\alpha \in(E X, A), \rho(\alpha)=e^{-1 / c}=\varphi(\bar{t}) e^{-\alpha \bar{t}}$ and $c \in\left(c_{0}, \infty\right)$.

5. $\sigma^{2}(t)>0$ and $\sigma^{2}(0)=E(X-E X)^{2}$.

6. $f(t)$ is a nondecreasing $(\nearrow)$ in $\left(0,1 / c_{0}\right), f(0)=0$ and $\lim _{t / t_{0}} f(t)=1 / c_{0}$.

7. $\xi(z)$ is a nondecreasing convex function such that $\xi(E X)=0$ and if $A<\infty$ then $\xi(z)=\infty$ for all $z>A$.

8. $\gamma(x)$ is a nondecreasing for $x<1 / c_{0}$, concave, if $c_{0}=0$ then $\gamma(x) \rightarrow \omega$ as $x \rightarrow \infty$ and if $c_{0}>0$ then $\gamma(x)=\omega$ for $x>1 / c_{0}$.

9. The functions $m(t), f(t), \xi(z)$ and $\gamma(x)$ satisfy the following relations:

$$
\begin{aligned}
& \xi(z)=f\left(m^{-1}(z)\right) \quad \text { for } \quad z \in[E X, A), \\
& \gamma(x)=m\left(f^{-1}(x)\right) \quad \text { for } \quad x \in\left[0,1 / c_{0}\right),
\end{aligned}
$$

where $m^{-1}(z)$ and $f^{-1}(x)$ are the inverse functions of $m(t)$ and $f(t)$ respectively.

\section{Remark 2.2.1.}

- If we take $a_{n}:=[c \log n]$ with $c>0$, then $b_{n}=[c \log n] \gamma(1 / c)$.

- Assume that the random variable satisfies condition $(H)$ together with $E X=0$ and $\sigma^{2}=E X^{2}<\infty$. Let $\frac{a_{n}}{\log n} \rightarrow \infty$. In this case, as $t \rightarrow 0$, the following holds

$$
\begin{gathered}
\varphi(t)=1+\frac{\sigma^{2} t^{2}}{2}(1+o(1)), \quad m(t)=\sigma^{2} t(1+o(1)) \\
\sigma^{2}(t)=\sigma^{2}(1+o(1)), \quad f(t)=\frac{\sigma^{2} t^{2}}{2}(1+o(1)) .
\end{gathered}
$$

From (??), (??) and (??), we get $\frac{\gamma(x)}{\sqrt{2 x \sigma^{2}}} \rightarrow 1$, as $x \rightarrow 0$. Hence, $b_{n} \sim \bar{b}_{n}$, where

$$
\bar{b}_{n}=\left(2 \sigma^{2} a_{n} \beta_{n}\right)^{1 / 2} .
$$

This $\bar{b}_{n}$ indicate the asymptotic formula of $b_{n}$ and will be used in few theorems in our thesis.

We have precise information about the constant $c_{0}$ defined by (??) in the following lemma. 
Lemma 2.2.2. For a given random variable $X$ satisfying condition $(H)$, we have $c_{0}=0$ in all cases except the following:

1. $A<\infty, t_{0}<\infty$. This covers all distributions with ess $\sup X<\infty$. In this case, $c_{0}=1 /\left(A t_{0}-\log \varphi\left(t_{0}\right)\right)$.

2. $A<\infty$, and $t_{0}=\infty$. This occurs if and only if ess $\sup X=\infty$. In this case, $A=e s s \sup X_{1}, P(X=A)>0$ and $c_{0}=-1 / \log \left(P\left(X_{1}=A\right)\right)$.

Proof: (see [?], p. 211)

The following examples explain the functions and the constants presented in formulas $(? ?)-(? ?)$.

Example 2.2.1. Let $X \sim N(0,1)$. Then $\varphi(t)=e^{t^{2} / 2}$,

$t_{0}=\sup \{t: \varphi(t)<\infty\}=\infty$

$m(t)=\frac{\varphi^{\prime}(t)}{\varphi(t)}=\frac{t e^{t^{2} / 2}}{e^{t^{2} / 2}}=t, \sigma^{2}(t)=m^{\prime}(t)=1, A=\lim _{t / t_{0}} m(t)=\infty$,

$f(t)=\operatorname{tm}(t)-\log \varphi(t)=\frac{t^{2}}{2}, \frac{1}{c_{0}}=\lim _{t / t_{0}} f(t)=\infty$, therefore $c_{0}=0$.

$$
\begin{gathered}
\xi(z)=\sup _{t \in \mathbb{R}}\{z t-\log \varphi(t): \varphi(t)<\infty\}=\sup _{t \geq 0}\left\{z t-\frac{t^{2}}{2}: \varphi(t)<\infty\right\}=\frac{z^{2}}{2} . \\
\rho(z)=e^{-z^{2} / 2} \text {, and } \gamma(x)=\sup \{z: \xi(z) \leq x\}=\sup \left\{z: z^{2} \leq 2 x\right\}=(2 x)^{1 / 2} .
\end{gathered}
$$

Example 2.2.2. Let $X \sim B(p)$, then $\varphi(t)=p e^{t}+1-p, w=\infty, t_{0}=\infty, m(t)=\frac{p e^{t}}{p e^{t}+1-p}$, $\sigma^{2}(t)=\frac{p e^{t}}{p e^{t}+1-p}-\left(\frac{p e^{t}}{p e^{t}+1-p}\right)^{2}, f(t)=t \frac{p e^{t}}{p e^{t}+1-p}-\log \left(p e^{t}+1-p\right), A=1, \frac{1}{c_{0}}=-\log p$, $\gamma(x)=\sup \{z: \xi(z)<x\}$, where $\xi(z)$ is given by;

$$
\xi(z)=\left\{\begin{array}{cc}
z \log \left(\frac{z}{p}\right)+(1-z) \log \left(\frac{1-z}{1-p}\right) & \text { if } 0 \leq z \leq 1 \\
\infty & \text { otherwise }
\end{array}\right.
$$

In this example observe that the function $\gamma(x)$ is difficult to compute. 


\section{Chapter 3}

\section{Strong Laws for Increments of Sums of i.i.d. Random Variables}

Beside the statistic

$$
\hat{D}_{n}\left(r_{n}\right)=\max _{0 \leq k \leq n-r_{n}}\left\{\left(k+r_{n}\right)\left(U_{k+r_{n}}(h)-\vartheta\right)-k\left(U_{k}(h)-\vartheta\right)\right\},
$$

we introduce functionals of this type or simply the following statistics:

$$
\begin{gathered}
\hat{T}_{n}\left(r_{n}\right)=\left(n+r_{n}\right)\left(U_{n+r_{n}}(h)-\vartheta\right)-n\left(U_{n}(h)-\vartheta\right), \\
\hat{R}_{n}\left(r_{n}\right)=n\left(U_{n}(h)-\vartheta\right)-\left(n-r_{n}\right)\left(U_{n-r_{n}}(h)-\vartheta\right), \\
\hat{W}_{n}\left(r_{n}\right)=\max _{0 \leq k \leq n-r_{n}} \max _{1 \leq j \leq r_{n}}\left\{(k+j)\left(U_{k+j}(h)-\vartheta\right)-k\left(U_{k}(h)-\vartheta\right)\right\},
\end{gathered}
$$

where $r_{n}$ is a nondecreasing sequence of natural numbers such that $m \leq r_{n} \leq n$.

- Note that, $\hat{D}_{n}(n)=\hat{R}_{n}(n)=n\left(U_{n}(h)-\vartheta\right)$ and $\hat{W}_{n}(n)=\max _{m \leq j \leq n} j\left(U_{j}(h)-\vartheta\right)$, if $a_{n}=n$.

- Let the kernel $h$ given by $h\left(x_{1}, x_{2}\right):=\frac{x_{1}+x_{2}}{2}$ and assume that $\vartheta:=E h\left(X_{1}, X_{2}\right)=0$. Then $\hat{D}_{n}\left(a_{n}\right)=\max _{2 \leq k \leq n-r_{n}}\left(S_{k+r_{n}}-S_{k}\right)$. Which is the same statistic as given by Erdös and Rényi [?].

The main goal of this chapter is to describe the a.s. asymptotic behavior of the statistics (??) - (??), but before doing that for the reader's convenience. We give an overview 
of the strong laws for increments of length $r_{n}$ for sums of independent and identically distributed random variables. We recall some well known results in this direction, which cover all sizes of increments with length $r_{n}$ such that $1 \leq r_{n} \leq n$. Among the authors, who studied these laws, we will focus our attention to the following ones:

- Erdös and Rényi [?], where they consider increments of length $r_{n}:=[c \log n]$, for positive number $c \geq c_{0}$.

- Book [?] extend [?] by considering increments of length $r_{n}:=(\log n)^{p}$, for $p>1$.

- Deheuvels and Devroye [?] consider increments of length $r_{n}:=[c \log n]$ for positive numbers $c \in\left(0, c_{0}\right)$ to cover all distributions characterized by Lemma ??.

- Csörgö and Révéz [?] extend [?] considering increments of length $(\log n)^{a} \leq r_{n} \leq n$, for some positive number $a>1$.

- Mason [?] extend [?] considering increments of length $1 \leq r_{n} \leq k_{n}$, where $k_{n}$ is a nondecreasing sequence of natural numbers satisfying $k_{n} / \log n \rightarrow 0$.

- Finally we mention Frolov [?, ?, ?], who considered increments of arbitrary length between 1 and $n$ with some restriction on the growth rate of $r_{n}$.

We will generalize these results to the case of U-statistics. In the first section of this chapter, almost sure behavior of short increments for sums of i.i.d. random variables are explained including the results by Erdös-Rényi and Shepp and their extensions given by Deheuvels et. al. [?], [?], Mason [?], and Book [?]. The second section includes the Csörgö-Révéz law [?]. The third section deals with a generalization to the case of arbitrary increments of length $r_{n}$ such that $1 \leq r_{n} \leq n$, given by Frolov [?, ?]. The chapter ends with establishing strong laws for increments, described by the statistics (??) - (??), of a nondegenerate U-statistics and some examples, which motivates our consideration.

\subsection{Erdös-Rényi and Shepp Laws}

Let us consider the Problem (A) given in formula (??) in the following special case: Consider a gambler who may play a sequence of games, in each of which he either wins or loses with probability $1 / 2$. For $j=1,2, \ldots$ interpret the event $X_{j}=1$ respectively $X_{j}=-1$ as the event that the gambler wins, respectively loses the $j t h$ game. Then, $D_{n}\left(r_{n}\right)$ describes the maximal gain over time subinterval of length $r_{n}$ and from the theory of sums of independent random variables, we obtain the following: 
1. Set in (??) $r_{n}=q_{n}=n$. We get by the strong law of large numbers that

$$
\lim _{n \rightarrow \infty} \frac{D_{n}\left(r_{n}\right)}{q_{n}}=\lim _{n \rightarrow \infty} \frac{1}{n} \sum_{j=1}^{n} X_{j}=E X_{1}=0 \text { a.s. }
$$

2. Set in (??) $r_{n}=q_{n}=1$. By Borel-Cantelli lemma we obtain that

$$
\lim _{n \rightarrow \infty} \frac{D_{n}\left(r_{n}\right)}{q_{n}}=\lim _{n \rightarrow \infty} \max _{1 \leq i \leq n} X_{i}=\text { ess } \sup X_{1}=1 \text { a.s. }
$$

3. Set in (??) $r_{n}=n$ and $q_{n}=\sqrt{2 n \log \log n}$. Thus, we get by the law of iterated logarithm (LIL) that

$$
\limsup _{n \rightarrow \infty} \frac{D_{n}\left(r_{n}\right)}{q_{n}}=\limsup _{n \rightarrow \infty} \frac{S_{n}}{\sqrt{2 n \log \log n}}=1 \text { a.s. }
$$

Hence assertion (??) tells us that in a long series $X_{1}, X_{2}, \ldots, X_{n}$ of trials, the empirical mean with probability one approximates the expected value of a single trial, which is 0 in our case. While assertion (??) tells us again and again that at least one trial is successful. Therefore, Problem(A) is reduced to the following problem: What will happen if one considers only subseries like $X_{k+1}+\ldots+X_{k+r_{n}}$ of length $r_{n}$, which may be short compared to the long series $X_{1}+X_{2}+\ldots+X_{n}$ ?

\subsubsection{Erdös-Rényi law}

Erdös and Rényi [?] answered Problem (A), and in fact they introduced the statistics $D_{n}\left(r_{n}\right)$ defined by formula (??). They proved that the maximal average gain is asymptotically between $E X_{1}=0$ and $\omega:=$ ess $\sup X_{1}=1$, which can be attained in a subseries of suitable length $r_{n}$ depending upon $n$ with probability one. They called their result as a new law of large numbers. More precisely, they proved that for positive number $c$,

$$
\lim _{n \rightarrow \infty} \frac{D_{n}([c \log n])}{[c \log n]}=\alpha \quad \text { a.s. }
$$

where $\alpha$ depending on $c$ and determined via the equation

$$
\rho(\alpha):=\inf _{t}\left\{\frac{e^{t}+e^{-t}}{2} e^{-t \alpha}\right\}=e^{-1 / c} .
$$

Note that if $\alpha \in\left(E X_{1}, \omega\right)$, then the above equation has a unique solution for $c \geq 1$. In fact the assertion (??) is a special form of the so-called Erdös-Rényi law, which is presented 
in the next theorem (see, Assumption (A) on p. 11).

Theorem 3.1.1. (Erdös-Rényi law for sums of i.i.d. random variables)

Consider a sequence $X_{1}, X_{2}, \ldots$ satisfying condition (H). Set $r_{n}=[c \log n]$. Then, for all $c>c_{0}$, we have:

$$
\lim _{n \rightarrow \infty} \frac{D_{n}([c \log n])}{[c \log n]}=\gamma(1 / c) \quad \text { a.s. }
$$

where $\gamma(1 / c)$ is defined in (??).

Under the same assumptions as in Theorem ?? the following statement holds:

$$
\lim _{n \rightarrow \infty} \frac{W_{n}([c \log n])}{[c \log n]}=\gamma(1 / c) \quad \text { a.s. }
$$

where $W_{n}\left(r_{n}\right)$ defined in (??). This was not given by Erdös and Rényi, but can be obtained in a similar way (see e.g., Steinebach [?]).

Remark 3.1.1. 1. The functional dependence between $c$ and $\alpha$ determines the distribution function of the random variable $X_{1}$, provided that the moment generating function of $X_{1}, \varphi(t)<\infty$ for some $|t|<t_{0}$. In fact, Erdös and Rényi were the first to point out such a connection between increments of processes and the underlying distribution in the case of partial sum sequences.

2. If $\varphi(t)=\infty$ for all $t>0$, then Steinebach [?] proved that for every positive constant $c$ we have

$$
\limsup _{n \rightarrow \infty} \frac{D_{n}([c \log n])}{[c \log n]}=\infty \text { a.s. }
$$

3. By virtue of Lemma ??, $c_{0}$ is not always 0, (see Example ??). Therefore, Theorem ?? gives us no information about the problem $(A)$ if $c \in\left(0, c_{0}\right)$. The answer of this problem was given by Deheuvels and Devroye [?]. Their results will be stated below.

4. Erdös and Rényi gave an application of their new law of large numbers to the socalled stochastic geyser problem.

Example 3.1.1. Let $\left\{X_{i}: i \geq 1\right\}$ be i.i.d. standard normally distributed random variables. Then $c_{0}=0$ and $\gamma(1 / c)=\sqrt{2 / c}$, (see Example ??). Hence, Theorem ?? assert that, for all $c>0$,

$$
\max _{0 \leq k \leq n-[c \log n]} \frac{S_{k+[c \log n]}-S_{k}}{[c \log n]} \rightarrow\left(\frac{2}{c}\right)^{1 / 2} \quad \text { a.s. }
$$


Example 3.1.2. Let $\left\{X, X_{1}, X_{2}, \ldots\right\}$ be i.i.d. Bernoulli trials with success probability $p=1 / 2$. Then $c_{0}=1 / \log (2)$ and Theorem ?? implies that, for all $c>1 / \log (2)$,

$$
\max _{0 \leq k \leq n-[c \log n]} \frac{S_{k+[c \log n]}-S_{k}}{[c \log n]} \rightarrow \gamma(1 / c) \quad \text { a.s. }
$$

We mentioned in Example ?? already that the computation of $\gamma(1 / c)$ is difficult, but using Erdös-Rényi Theorem, we can estimate it with probability one by the ratio $\frac{D_{n}([c \log n])}{[c \log n]}$, which is of interest from the large deviations point of view.

Theorem ?? has an extension to nondegenerate U-statistics by an application of Corollary ??. However we only are able to extend Theorem ?? for arbitrary U-statistics with kernel $h$ of degree $m=2$ which is given in Theorem ??. We give also the upper limit for arbitrary kernel $h$ of degree $m \geq 2$ in Lemma ??. The same thing can be said for Theorem ??, which will be stated below.

\subsubsection{Shepp law}

In 1964 Shepp introduced the statistics,

$$
T_{n}(f(n))=\left(S_{n+f(n)}-S_{n}\right),
$$

where he developed $\lim \sup _{n \rightarrow \infty} \frac{T_{n}(f(n))}{f(n)}$ in terms of the moment generating function $\varphi(t)$ and the radius of convergence of $\sum x^{f(n)}$, denoted by $\mathrm{R}$.

Theorem 3.1.2. (Shepp law for sums of i.i.d. random variables)

Let $X_{1}, X_{2}, \ldots$, be a sequence of i.i.d. random variables such that $\varphi(t)<\infty$ for some $t>0$. Let $f(n)$ be a nondecreasing sequence of positive integer values tending to infinity. Then we have

$$
\limsup _{n \rightarrow \infty} \frac{T_{n}(f(n))}{f(n)}=\left\{\begin{array}{cc}
\omega & \text { if } 0 \leq R \leq P\left(X_{1}=\omega\right) \\
\alpha_{R} & \text { if } P\left(X_{1}=\omega\right)<R<1 \\
E X_{1} & \text { if } R=1,
\end{array}\right.
$$

where $\alpha:=\alpha_{R}$ is the unique solution of the equation $\rho(\alpha)=R$ for $\alpha \in\left(E X_{1}, \omega\right)$ and $\omega:=e s s \sup X_{1}$.

Considering the sequence $-X_{i}$ leads to the analogue theorem with lim inf replaced by limsup. If $f(n)$ is strictly increasing then $R=1$. The existence of $\lim _{n \rightarrow \infty} T_{n}(f(n))$ is assumed if and only if $R=1$, whence $\lim _{n \rightarrow \infty} T_{n}(f(n))=E X_{1}$, a.s., $-\infty<E X_{1}<\infty$.

Remark 3.1.2. 1. If we take $f(n):=[c \log n], c>0$ in Theorem ??, then $R=e^{-1 / c}$. 
2. If $\varphi(t)=\infty$ for all $t>0$, then Lynch [?] proved that $\lim \sup _{n \rightarrow \infty} \frac{T_{n}(f(n))}{f(n)}=\infty$, for all subsequences $f(n)$ for which $R<1$.

Example 3.1.3. Let $\left\{X, X_{1}, X_{2}, \ldots\right\}$ be i.i.d. Bernoulli trials with success probability $p \in(0,1)$. Then

$$
\rho(\alpha):=\min _{t} \varphi(t) e^{-t \alpha}=\left(\frac{p}{\alpha}\right)^{\alpha}\left(\frac{1-p}{1-\alpha}\right)^{1-\alpha}
$$

Hence, Shepp law guarantees that

$$
\limsup _{n \rightarrow \infty} \frac{T_{n}(f(n))}{f(n)}=\left\{\begin{array}{cl}
\alpha & \text { for } p \leq R \leq 1 \\
1 & \text { for } 0 \leq R \leq p
\end{array}\right.
$$

where $\alpha$ is the unique solution of the equation, $R=\left(\frac{p}{\alpha}\right)^{\alpha}\left(\frac{1-p}{1-\alpha}\right)^{1-\alpha}$. Set $f(n):=[c \log n]$ and $p=1 / 2$, then Theorem ?? together with Remark ?? implies that,

$$
\limsup _{n \rightarrow \infty} \frac{T_{n}([c \log n])}{[c \log n]}=\left\{\begin{array}{cc}
\alpha & \text { for } c>1 / \log (2) \\
1 & \text { for } 0<c \leq 1 / \log (2)
\end{array}\right.
$$

where $\alpha$ is the unique solution of the equation $e^{-1 / c}=\frac{1}{2}\left(\frac{1}{\alpha}\right)^{\alpha}\left(\frac{1}{1-\alpha}\right)^{1-\alpha}$.

\subsubsection{Full form of Erdös-Rényi law}

In Remark ??, we observed that Theorem ?? provides no information about the asymptotic behavior of $D_{n}([c \log n])$ when $c \in\left(0, c_{0}\right)$. Also the asymptotic behavior for the statistics $W_{n}([c \log n])$ is not included in that theorem. In this section, we discuss the asymptotic behavior of $D_{n}([c \log n])$ and $W_{n}([c \log n])$, when $c \in\left(0, c_{0}\right)$. This corresponds to the so-called full form of Erdös-Rényi law due to Deheuvels and Devroye [?].

The following theorem due to Deheuvels and Devroye (see, [?], p. 1374), which covers specific distributions characterized in Lemma ??.

Theorem 3.1.3. Consider a sequence $X_{1}, X_{2}, \ldots$ that satisfies condition (H). Set $r_{n}=$ $[c \log n]$ for $0<c \leq c_{0}$. Then we have

$$
\lim _{n \rightarrow \infty} \frac{D_{n}([c \log n])}{[c \log n]}=\lim _{n \rightarrow \infty} \frac{W_{n}([c \log n])}{[c \log n]}=A+\frac{1}{t_{0}}\left(\frac{1}{c}-\frac{1}{c_{0}}\right) \quad \text { a.s. },
$$

where $c_{0}$ and $A$ are defined in (??).

Theorem ?? has an extension to nondegenerate U-statistics with kernel $h$ of degree $m \geq 2$ by an application of Corollary ??. 


\subsubsection{Extended Erdös-Rényi law}

Let $X_{1}, X_{2}, \ldots$ be a sequence of i.i.d. $\mathrm{N}(0,1)$ distributed random variables. Then $E e^{t X_{1}}<$ $\infty$ for all $t \in \mathbb{R}$. For $c<1 / 2$, we have $E e^{c X_{1}^{2}}<\infty$, and $E e^{c X_{1}^{2}}=\infty$, for $c \geq 1 / 2$. Consequently, for $c \geq 1 / 2$ we get $e^{X_{1}^{2} / 2}>n$ infinitely often. Hence, $\left|X_{n}\right|>\sqrt{2} \log n$ infinitely. Thus $\lim \sup \frac{X_{n}}{\sqrt{2 \log n}} \geq 1$. On the other hand, put $c=1 / 2-\epsilon$, where we choose $\epsilon$ to be a very small number. Then we eventually obtain $\left|X_{n}\right| \leq \sqrt{2.001 \log n}$. Thus, $\lim \sup \frac{X_{n}}{\sqrt{2 \log n}} \leq 1.001$. Hence, $\lim \sup \frac{X_{n}}{\sqrt{2 \log n}}=1$.

In 1989 Mason [?] extended the Erdös and Rényi Theorem ?? considering increments of length $r_{n}$ in the definition of the statistic $D_{n}\left(r_{n}\right)$ such that $c(n):=\frac{\log n}{r_{n}} \rightarrow \infty$. He found a norming sequence $q_{n}$, which depends on the full distribution of $X_{1}$. His theorem (see [?]) makes the following assertion.

Theorem 3.1.4. (Extended Erdös-Rényi law for sums of i.i.d. random variables) Let $X_{1}, X_{2}, \ldots$ be a sequence of i.i.d. random variables satisfying condition (H).

(a) If $0<\omega<\infty$, then for all sequences $r_{n}$ such that $c(n)=\frac{\log n}{r_{n}} \rightarrow \infty$,

$$
\lim _{n \rightarrow \infty} \max _{0 \leq k \leq n-r_{n}} \frac{S_{k+r_{n}}-S_{k}}{r_{n} \gamma(c(n))}=\lim _{n \rightarrow \infty} \max _{0 \leq k \leq n-r_{n}} \frac{S_{k+r_{n}}-S_{k}}{\omega r_{n}}=1 \text { a.s. }
$$

(b) If $\omega=\infty$, then for all sequences $c(n)$ such that $c(n)=\frac{\log n}{r_{n}} \rightarrow \infty$, we have

$$
\limsup _{n \rightarrow \infty} \max _{0 \leq k \leq n-r_{n}} \frac{S_{k+r_{n}}-S_{k}}{\gamma(c(n)) r_{n}}=1 \quad \text { a.s. }
$$

Moreover, limsup in (b) can be replaced by $\lim$ for all such sequences $r_{n}$ if and only if

$$
\lim _{x \rightarrow \infty} \frac{\gamma(-\log (1-F(x)))}{x}=1
$$

if and only if

$$
\lim _{n \rightarrow \infty} \max _{1 \leq m \leq n} \frac{X_{m}}{\gamma(\log n)}=1 \quad \text { a.s. }
$$

which in turn is equivalent to (??) with $r_{n}=1$.

Remark 3.1.3. Some examples of distributions which satisfy (??) are normal, geometric, poisson and Weibull with shape parameter $a \geq 1$.

Theorem ?? has an extension to nondegenerate U-statistics by an application of Corollary ?? partly. However we are only able to give the upper limit for arbitrary U-statistics 
with kernel $h$ of degree $m \geq 2$ which is given in Lemma ?? or Lemma ??.

Book proved various generalizations of the Erdös-Rényi law for weighted sum and nonidentically distributed random variables. Among them we state the following theorem due to Book in 1975, which arises from the extended strong law of large number given by Marcinkiewics and Zygmund, where increments or blocks of length approximately $(c \log n)^{p}$ for $p>1$ are considered.

Theorem 3.1.5. (Generalized Erdös-Rényi law for sums of i.i.d. random variables) Let $X_{1}, X_{2}, \ldots$ be a sequence of i.i.d. random variables satisfying $E X_{1}=0$ and $\operatorname{Var}\left(X_{1}\right)=$ 1. Assume that $\varphi(t)<\infty$ for $|t|<B$, where $0<B \leq \infty$. Let $1<p<2, \beta:=(2-p) / p$ and for every $\alpha>0$, set $r_{n}:=\left[\left(\frac{2}{\alpha^{2}} \log n\right)^{1 / \beta}\right]$. Then we have

$$
\frac{D_{n}\left(r_{n}\right)}{r_{n}^{1 / p}} \rightarrow \alpha \quad \text { a.s. }
$$

1. The constant $2 \alpha^{-2}$ depends only on $\alpha$ and is independent of the distribution of $X_{1}$ contrary to Erdös and Rényi result, where $\alpha$ depends on the distribution of $X_{1}$.

2. If $X_{1} \sim N(0,1)$, then the conclusion of Theorem (??) holds for $1 \leq p<2$, since by Theorem ??, for every $\alpha>0$, we have $c=2 / \alpha^{2}$.

We give two extensions of Theorem ?? to U-statistics. Theorem ?? for general kernels and Theorem ?? for product kernels. These theorems are proved by an application of Corollary ??. In fact we are only able to prove these results for nondegenerate U-statistics. However we give the upper limit for arbitrary U-statistics with kernel $h$ of degree $m \geq 2$ which is given in Lemma ??.

\subsection{Csörgö -Révéz Law}

In this section we consider the asymptotic behavior of statistics (??) - (??), when the increments are large. Using analogous results for increments of a Wiener processes and the Komlós-Major-Tusnády strong approximation (see [?], p. 107) Csörgö and Révéz [?] extend the Erdös-Rényi law for sums of i.i.d. random variables considering large increments.

Theorem 3.2.1. (Csörgö-Révéz Law)

Let $W(t), 0 \leq t<\infty$ be a standard Wiener process. Let $a_{T}, T \geq 0$, be a nondecreasing function of $T$ for which 
1. $0<a_{T} \leq T$,

2. $T / a_{T}$ is nondecreasing.

are satisfied. Then

$\limsup _{T \rightarrow \infty} \sup _{0 \leq t \leq T-a_{T}} \frac{1}{\bar{b}_{T}}\left|W\left(t+a_{T}\right)-W(t)\right|=\limsup _{T \rightarrow \infty} \sup _{0 \leq s \leq a_{T}} \frac{1}{\bar{b}_{T}}|W(T+s)-W(T)|=1 \quad$ a.s., and

$\limsup _{T \rightarrow \infty} \sup _{0 \leq t \leq T-a_{T}} \sup _{0 \leq s \leq a_{T}} \frac{1}{\bar{b}_{T}}|W(t+s)-W(t)|=\limsup _{T \rightarrow \infty} \frac{1}{\bar{b}_{T}}\left|W\left(T+a_{T}\right)-W(T)\right|=1 \quad$ a.s. , where $\bar{b}_{T}=\left(2 a_{T}\left(\log \left(T / a_{T}\right)+\log \log T\right)\right)^{1 / 2}$. If additionally $a_{T}$ satisfies

$$
\lim _{T \rightarrow \infty} \frac{\log \left(T / a_{T}\right)}{\log \log T}=\infty
$$

then

$\lim _{T \rightarrow \infty} \sup _{0 \leq t \leq T-a_{T}} \frac{1}{\bar{b}_{T}}\left|W\left(t+a_{T}\right)-W(t)\right|=\lim _{T \rightarrow \infty} \sup _{0 \leq t \leq T-a_{T}} \sup _{0 \leq s \leq a_{T}} \frac{1}{\bar{b}_{T}}|W(t+s)-W(t)|=1 \quad$ a.s.

\section{Remark 3.2.1.}

1. By symmetry of $W$, if we replace limsup by liminf and sup by inf in Theorem ??, then the assertion above will be true with -1 instead of 1.

2. Choosing $a_{T}$ as $c \log T$ and $d T$ for $0<d \leq 1$, we get Erdös-Rényi law and Strassen's law of iterated logarithm for the standard Wiener process respectively.

3. Let $X_{1}, X_{2}, \ldots$ be i.i.d. random variables satisfying $E X_{1}=1, \operatorname{Var}\left(X_{1}\right)=1$ and $E e^{t X}<\infty$, for $|t|<t_{0}$. Then Theorem ?? and the strong invariance principle of Komlós-Major-Tusnády imply the so-called Csörgö-Révéz law for sums of i.i.d. random variables, i.e, with probability one the following holds.

$$
\limsup _{n \rightarrow \infty} \frac{D_{n}\left(a_{n}\right)}{\bar{b}_{n}}=\limsup _{n \rightarrow \infty} \frac{W_{n}\left(a_{n}\right)}{\bar{b}_{n}}=\limsup _{n \rightarrow \infty} \frac{R_{n}\left(a_{n}\right)}{\bar{b}_{n}}=\limsup _{n \rightarrow \infty} \frac{T_{n}\left(a_{n}\right)}{\bar{b}_{n}}=1,
$$

and (??) will be true, if we replace limsup by liminf, sup by inf and 1 by -1 .

If additionally $a_{n}$ satisfies

$$
\lim _{n \rightarrow \infty} \frac{\log \left(n / a_{n}\right)}{\log \log n}=\infty
$$


then

$$
\lim _{n \rightarrow \infty} \frac{D_{n}\left(a_{n}\right)}{\bar{b}_{n}}=\lim _{n \rightarrow \infty} \frac{W_{n}\left(a_{n}\right)}{\bar{b}_{n}}=1 \quad \text { a.s. }
$$

Steinebach [?] and Lynch [?] proved that if the moment generation function $\varphi(t)=\infty$ for all $t>0$, then the strong laws for short increments of sums of i.i.d. random variables are not more available. On the contrary, Csörgö and Révéz present a theorem analogues to Theorem ?? in the case of large increments assuming only the existence of a finite number of moments of $X_{1}$. Instead of mentioning their results, we state its generalization given by Frolov [?].

\subsubsection{One-Sided Strong Laws for Increments of Sums of i.i.d. Random Variables}

Frolov [?] studied the asymptotic behavior of statistics given in formulas (??) - (??) of large increments when the trajectories of sum $S_{n}$ have large jumps only in one direction. It arises, for example, for the case where the random variable $X_{1}$ is in the domain of attraction of a completely asymmetric stable law. Then (??) and the other case do not hold together, rather only one of them can hold. In this case, $S_{n}$ can not be well approximated by a wiener process but large deviations techniques are used. His theorem make the following assertion.

Theorem 3.2.2. Let $\left\{X, X_{1}, X_{2}, \ldots\right\}$ be i.i.d. random variables with $E X=0$ and $\operatorname{Var}(X)=1$. Set $r_{n}=a_{n}$, where $a_{n}$ satisfies the assumptions (A). Suppose that one of the following two conditions holds:

1. $\int_{0}^{\infty} e^{t x^{b}} d F(x)<\infty$, for some $t>0$ and $0<b \leq 1$, and $\frac{a_{n}}{(\log n)^{\frac{2}{b}-1}} \rightarrow \infty$ as $n \rightarrow \infty$.

2. $\int_{0}^{\infty} x^{p} d F(x)<\infty$, for some $p>2, \quad \log n \int_{-\infty}^{-n} x^{2} d F(x) \rightarrow 0$, and $a_{n}>\frac{c n^{2 / p}}{\log n}>0$ for some $c>0$ and all large $n$.

Then we have

$$
\limsup _{n \rightarrow \infty} \frac{D_{n}\left(a_{n}\right)}{\bar{b}_{n}}=\limsup _{n \rightarrow \infty} \frac{W_{n}\left(a_{n}\right)}{\bar{b}_{n}}=\limsup _{n \rightarrow \infty} \frac{\tilde{R}_{n}\left(a_{n}\right)}{\bar{b}_{n}}=\limsup _{n \rightarrow \infty} \frac{T_{n}\left(a_{n}\right)}{\bar{b}_{n}}=1 \quad \text { a.s. }
$$

If in addition $\lim _{n \rightarrow \infty} \frac{\log \left(\frac{n}{a_{n}}\right)}{\log \log n}=\infty$ is satisfied, then

$$
\lim _{n \rightarrow \infty} \frac{D_{n}\left(a_{n}\right)}{\bar{b}_{n}}=\lim _{n \rightarrow \infty} \frac{W_{n}\left(a_{n}\right)}{\bar{b}_{n}}=1 \text { a.s. }
$$


Proof: (see [?])

Remark 3.2.2. Let the assumptions of Theorem ?? are satisfied for both $X_{i}$ and $-X_{i}$. If we replace limsup by liminf and sup by inf in the definition of statistics given by (??) - (??), then the assertion of Theorem ?? holds true with -1 instead of 1.

Theorem ?? has an extension to the statistics (??) - (??), where only nondegenerate U-statistics with kernel $h$ of degree $m \geq 2$ are considered. This is given in Corollary ??. In Lemma ?? we extend Theorem ?? partly, where we obtain the lower limit for the statistics (??) - (??) considering nondegenerate U-statistics with kernel $h$ of degree $m \geq 2$. We also obtain the upper limit for the statistics (??) and (??) considering arbitrary U-statistics in Lemma ??.

\subsection{Strong Laws for Increments of Sums of i.i.d. Ran- dom Variables}

Frolov [?] found a universal formula for the norming sequence in strong limit theorems for increments of sums of i.i.d. random variables, provided that the random variable $X_{1}$ satisfying the condition $(\mathrm{H})$. By this formula, we can calculate norming sequences in SLLN, LIL, Erdös-Rényi and Shepp law, and Csörgö -Révéz law. This norming sequence denoted by $b_{n}$ and given in (??), where $\gamma(x)$ is defined by (??).

Depending on the constants $t_{0}, A$ and $c_{0}$, there are five classes of distributions (see, assumption (A) on p. 13):

- (I) $t_{0}=\infty, A<\infty, c_{0}=0$. In this case $\omega=A$.

- (II) $t_{0}=\infty, A<\infty, c_{0}>0$. In this case $\omega=A, c_{0}=-1 / \log P(X=A)$.

- (III) $t_{0}=\infty, A=\infty$. In this case $\omega=\infty, c_{0}=0$.

- (IV) $t_{0}<\infty, A=\infty$. In this case $\omega=\infty, c_{0}=0$.

- (V) $t_{0}<\infty, A<\infty$. In this case $\omega=\infty, c_{0}=\left(t_{0} A-\log \varphi\left(t_{0}\right)\right)^{-1}>0$.

For example, the uniform, Bernoulli, normal, exponential and the distribution with density function $p(x)=C x^{-3} e^{-x}, x \geq 1$ belong to the classes I-V respectively.

The following theorem is due to Frolov [?], which contain all of the preceding results (Erdös-Rényi Theorem ??, Shepp Theorem ??, Deheuvels and Devroye Theorem ??, part of Mason Theorem ?? and Frolov Theorem ??) as special case. 
Theorem 3.3.1. Let $X, X_{1}, X_{2}, \ldots$ be a sequence of i.i.d. random variables satisfying condition (H).

A) Assume that there exists a function $g(\theta), \theta \in\left(1, \theta_{0}\right)$, such that $g(\theta) \rightarrow 1$ as $\theta \rightarrow 1$ and $\frac{b(x \theta)}{b(x)} \leq g(\theta)$ for all sufficiently large $x$. Suppose that one of the following two conditions is satisfied:

(i) $\frac{\log a_{n}}{\log n} \rightarrow 0$.

(ii) For every $\epsilon>0$, there exists $q \in(0,1)$ such that

$$
P\left(S_{i} \geq-\epsilon b_{n}\right) \geq q
$$

holds for all $i \leq I_{n}:=\min \left\{\left[(1+\epsilon) a_{n}\right], n\right\}$ and for all sufficiently large $n$. Then

$$
\limsup _{n \rightarrow \infty} \frac{W_{n}\left(a_{n}\right)}{b_{n}} \leq 1 \quad \text { a.s. }
$$

B) Further for $\epsilon \in(0,1)$, set

$$
t^{\star}=f^{-1}\left(\frac{1-\epsilon}{a_{n}}\left(\log \frac{n}{a_{n}}+\log \log n\right)\right),
$$

and assume that one of the following conditions is satisfied:

1.) $t^{\star}<t_{0}$ and for all sufficiently small $\epsilon \in(0,1)$,

$$
t^{\star} \sigma\left(t^{\star}\right)=o\left(\left(a_{n}\right)^{1 / 2} f\left(t^{\star}\right)\right)
$$

2.) $F \in K_{I}$ and $t^{\star} \rightarrow \infty$

3.) $F \in K_{I I}$ and $\frac{\log \left(n / a_{n}\right)+\log \log n}{a_{n}} \geq 1 / c_{0}$

4.) $F \in K_{I I I}$ or $F \in K_{V}, t^{\star} \rightarrow \infty$ and $\forall \tau>0, \delta>0$ and all sufficiently large t;

$$
P(X \geq(1-\tau) m(t)) \geq \exp \{-(1+\delta) f(t)\}
$$

5.) $F \in K_{I V}, t^{\star} \nearrow t_{0}$ and the inequality (??) holds $\forall \tau>0, \delta>0$ and for all $t$ sufficiently close to $t_{0}$;

6.) $F \in K_{V}, t^{\star} \geq t_{0}$ and $t^{\star}$ does not depend on $n$.

Then,

$$
\limsup _{n \rightarrow \infty} \frac{R_{n}\left(a_{n}\right)}{b_{n}} \geq 1 \quad \text { a.s. }
$$

C) Furthermore, if two conditions, one from A) and the other from B), are satisfied, 
then a.s.,

$$
\limsup _{n \rightarrow \infty} \frac{W_{n}\left(a_{n}\right)}{b_{n}}=\limsup _{n \rightarrow \infty} \frac{D_{n}\left(a_{n}\right)}{b_{n}}=\limsup _{n \rightarrow \infty} \frac{R_{n}\left(a_{n}\right)}{b_{n}}=\limsup _{n \rightarrow \infty} \frac{T_{n}\left(a_{n}\right)}{b_{n}}=1 .
$$

If, in addition, $\log \log n=o\left(\log \frac{n}{a_{n}}\right)$, then

$$
\lim _{n \rightarrow \infty} \frac{W_{n}\left(a_{n}\right)}{b_{n}}=\lim _{n \rightarrow \infty} \frac{D_{n}\left(a_{n}\right)}{b_{n}}=1 \quad \text { a.s. }
$$

Proof: (see [?])

Remark 3.3.1. 1. If $\left|I_{n+1}-I_{n}\right| \leq C$, for a constant $C$ and for all sufficiently large $n$ and $b_{n} \rightarrow \infty$, then it is sufficient to check condition (??) just for $i=I_{n}$. In particular, if $a_{n}=[a(n)]$, then $\left|I_{n+1}-I_{n}\right| \leq 3$.

2. If the distribution function of $X$ belongs to class $K_{I}$ or $K_{I I}$ and $\log a_{n}=o(\log n)$, then the assertion $(A)$ of the theorem becomes trivial by noting that $\omega<\infty$ and $b_{n} \sim \omega a_{n}$.

\subsection{Strong Laws for Increments for Nondegenerate U-Statistics}

In this section, we establish the strong laws for increments, described by the statistics (??) - (??), of a nondegenerate U-statistics. The strong laws for increments of sums of i.i.d. random variables discussed in the last three sections can be summarizes in two results due to Frolov [?, ?, ?]. They are Theorem ?? if the moment generating function does not exists and Theorem ?? if moment generating function exists in a right neighborhood of zero. Therefore we focus our attention to generalize Theorem ?? and Theorem ?? to the statistics (??) - (??). To that aim we use H-decomposition Theorem ??, i.e. we write

$$
U_{n}(h)-\vartheta=m U_{n}\left(h_{1}\right)+R_{n}^{*}
$$

where $R_{n}^{*}:=\sum_{d=2}^{m}\left(\begin{array}{c}m \\ d\end{array}\right) U_{n}\left(h_{d}\right)$. It is already known that $R_{n}^{*}$ is a reverse martingale (see e.g., [?] p. 188). More precise our idea is to rewrite the statistics (??) - (??) in the form (??) and prove that $R_{n}^{*}$ normalized by appropriate sequence of positive numbers has no effect on the asymptotic behavior of them with probability 1. For example, let us consider 
the statistics $\hat{T}_{n}\left(a_{n}\right)$ :

$$
\begin{aligned}
& \hat{T}_{n}\left(a_{n}\right):=\left(n+a_{n}\right)\left(U_{n+a_{n}}(h)-\vartheta\right)-n\left(U_{n}(h)-\vartheta\right) \\
& =\left(n+a_{n}\right) m U_{n+a_{n}}\left(h_{1}\right)+\left(n+a_{n}\right) \sum_{d=2}^{m}\left(\begin{array}{c}
m \\
d
\end{array}\right) U_{n}\left(h_{d}\right)-m n U_{n}\left(h_{1}\right)-n \sum_{d=2}^{m}\left(\begin{array}{c}
m \\
d
\end{array}\right) U_{n}\left(h_{d}\right) \\
& =m \sum_{i=1}^{n+a_{n}} h_{1}\left(X_{i}\right)+\left(n+a_{n}\right) R_{n+a_{n}}^{*}-m \sum_{i=1}^{n} h_{1}\left(X_{i}\right)-n R_{n}^{*} \\
& =m \sum_{i=n+1}^{n+a_{n}} h_{1}\left(X_{i}\right)+\left(n+a_{n}\right) R_{n+a_{n}}^{*}-n R_{n}^{*}
\end{aligned}
$$

Note that the first term in (??) is a sum of i.i.d. random variables, therefore we can used the previous results for our aim.

Throughout this section, we say that a kernel $h: \mathbb{R}^{m} \rightarrow \mathbb{R}$ satisfy condition (H1), if it satisfies

1. $h$ is nondegenerate, i.e, $\zeta_{1}^{2}:=\operatorname{Var}\left(h_{1}\left(X_{i}\right)\right)>0$.

2. $t_{0}:=\sup \left\{t: \phi(t)=E e^{t h_{1}(X)}<\infty\right\}>0$.

3. $E h^{2}<\infty$.

For the kernel $h$ satisfying the condition (H1) and $t \leq t_{0}$, we define the collection of constants and functions

$$
\varphi_{h_{1}}(t), m_{h_{1}}(t), \sigma_{h_{1}}^{2}(t), f_{h_{1}}(t), \omega_{h_{1}}, \xi_{h_{1}}(\alpha) \text { and } \gamma_{h_{1}}(x),
$$

by (??) - (??) with $X$ replaced by $h_{1}\left(X_{1}\right)$.

We are now in the position to generalize Theorem ?? applied to U-statistics, with other words, the following corollary describe the a.s. asymptotic behavior of the statistics (??) - (??) depending on the growth rate of the sequence $a_{n}$.

Corollary 3.4.1. Consider a nondegenerate U-statistics $U_{n}(h)$ based on a kernel $h$ of degree $m$ satisfying the condition (H1). Assume that the assumptions of Theorem ?? with $X$ replaced by $h_{1}\left(X_{1}\right)$ are satisfied. Then

$$
\limsup _{n \rightarrow \infty} \frac{\hat{W}_{n}\left(a_{n}\right)}{\hat{b}_{n}}=\limsup _{n \rightarrow \infty} \frac{\hat{D}_{n}\left(a_{n}\right)}{\hat{b}_{n}}=\limsup _{n \rightarrow \infty} \frac{\hat{R}_{n}\left(a_{n}\right)}{\hat{b}_{n}}=\limsup _{n \rightarrow \infty} \frac{\hat{T}_{n}\left(a_{n}\right)}{\hat{b}_{n}}=1 \text { a.s. }
$$


If in addition, $\log \log n=o\left(\log \frac{n}{a_{n}}\right)$, then

$$
\lim _{n \rightarrow \infty} \frac{\hat{W}_{n}\left(a_{n}\right)}{\hat{b}_{n}}=\lim _{n \rightarrow \infty} \frac{\hat{D}_{n}\left(a_{n}\right)}{\hat{b}_{n}}=1 \quad \text { a.s. },
$$

where $\hat{b}_{n}:=a_{n} \gamma_{h_{1}}\left(\frac{\beta_{n}}{a_{n}}\right)$.

Proof: The proof follows by Theorem ?? and the following Proposition ?? (given below) via the representation (??).

Proposition 3.4.1. Suppose that the kernel $h$ satisfies $E h^{2}\left(X_{1}, \ldots, X_{m}\right)<\infty$. Then

$$
\frac{n}{\hat{b}_{n}} R_{n}^{*} \rightarrow 0 \quad \text { a.s. }
$$

with appropriate choice of $a_{n}$.

Proof: Since $R_{n}^{*}$ is a reverse martingale and the sequence $n / \hat{b}_{n}$ is non-decreasing, it follows by using Lemma ?? that

$$
\begin{aligned}
P\left(\sup _{n \geq p} \frac{n}{\hat{b}_{n}}\left|R_{n}^{*}\right|>\epsilon\right) & \leq \epsilon^{-2} \sum_{n=p}^{\infty} \frac{n^{2}}{\left(\hat{b}_{n}\right)^{2}} E\left(R_{n}^{* 2}-R_{n+1}^{* 2}\right) \\
& <C \sum_{n=p}^{\infty} \frac{n^{2}}{\left(\hat{b}_{n}\right)^{2}} n^{-3}=C \sum_{n=p}^{\infty} \frac{1}{n\left(\hat{b}_{n}\right)^{2}}
\end{aligned}
$$

which converge by appropriate choice of $a_{n}$ as $p \rightarrow \infty$, hence the statement follows by Borel-Canteli lemma.

Notice that for a suitable choice of $a_{n}$ the relation (??) implies that

$$
\max _{0 \leq k \leq n-a_{n}} \frac{k}{\hat{b}_{n}} R_{k}^{*} \rightarrow 0 \quad \text { a.s., as } n \rightarrow \infty \text {. }
$$

\subsubsection{Application of Corollary ?? to Strong Limit Theorem of U-Statistics}

- Consider the increments given by Erdös-Rényi and Shepp, i.e. $a_{n}=[c \log n]$ with $c>$ 0 , then $\hat{b}_{n}=[c \log n] \gamma_{h_{1}}(1 / c)$ and therefore the series in Proposition ?? converges. Since $\frac{\log [c \log n]}{\log n} \rightarrow 0$, the condition $(i)$ of Theorem ?? related to Corollary ?? is 
satisfied. Furthermore for $c>c_{0}$, we have

$$
\frac{1-\epsilon}{a_{n}}\left(\log \frac{n}{a_{n}}+\log \log n\right)<\frac{1-\epsilon}{c}<\frac{1}{c_{0}}, \text { for all } c>c_{0},
$$

then by Lemma ??, $f_{h_{1}}^{-1}$ exists in $\left(0,1 / c_{0}\right)$ and $t^{\star} \leq f_{h_{1}}^{-1}\left(\frac{1-\epsilon}{c}\right)<t_{0}$. Note also

$$
t^{\star} \sigma_{h_{1}}\left(t^{\star}\right)=f_{h_{1}}^{-1}\left(\frac{1-\epsilon}{c}\right) \sigma_{h_{1}}\left(f_{h_{1}}^{-1}\left(\frac{1-\epsilon}{c}\right)\right)=o\left(\sqrt{a_{n}} f_{h_{1}}\left(t^{\star}\right)\right) .
$$

Hence condition 1.) of Theorem ?? related to Corollary ?? is satisfied. If $c<c_{0}$, then we need first to know about the type of the distribution function of $h_{1}\left(X_{1}\right)$ to check the suitable condition of Theorem ?? related to Corollary ??. By observing that $\log \log n=o\left(\log \frac{n}{a_{n}}\right)$ Corollary ?? implies

$$
\lim _{n \rightarrow \infty} \frac{\hat{W}_{n}([c \log n])}{[c \log n]}=\lim _{n \rightarrow \infty} \frac{\hat{D}_{n}([c \log n])}{[c \log n]}=\limsup _{n \rightarrow \infty} \frac{\hat{T}_{n}([c \log n])}{[c \log n]}=m \gamma_{h_{1}}(1 / c) \quad \text { a.s. }
$$

Which may be called the full form of the Erdös-Rényi and Shepp laws for nondegenerate U-statistics.

- Consider the increments given by Csörgö- Révéz, i.e. $a_{n}$ satisfy the relation, $\frac{a_{n}}{\log n} \rightarrow$ $\infty$. Then to calculate the norming sequence $\hat{b}_{n}$, we observe that $\frac{\beta_{n}}{a_{n}} \rightarrow 0$. Therefore we calculate the asymptotic formula of $\hat{b}_{n}$. As $t \rightarrow 0$, the following holds true:

$$
\begin{gathered}
\varphi_{h_{1}}(t)=1+\frac{\zeta_{1}^{2} t^{2}}{2}(1+o(1)), \quad m_{h_{1}}(t)=\zeta_{1}^{2} t(1+o(1)) \\
\sigma_{h_{1}}^{2}(t)=\zeta_{1}^{2}(1+o(1)), \quad f_{h_{1}}(t)=\frac{\zeta_{1}^{2} t^{2}}{2}(1+o(1))
\end{gathered}
$$

In this case condition 1.) of Theorem ?? related to Corollary ?? is satisfied. From (??), (??) and the definition of $\gamma_{h_{1}}(x)$, we obtain

$$
\frac{\gamma_{h_{1}}(x)}{\sqrt{2 x \zeta_{1}^{2}}} \rightarrow 1 \quad \text { as } x \rightarrow 0 .
$$

Thus

$$
\hat{b}_{n}=\left(2 \zeta_{1}^{2} a_{n}\left(\log \left(n / a_{n}\right)+\log \log n\right)\right)^{1 / 2} .
$$

Now it is easy to see that the series in Proposition ?? converges. In fact the condition (ii) of Theorem ?? related to Corollary ?? is satisfied. Indeed using Chebyshev's 
inequality to get for all $\epsilon>0$ and $I_{n}:=\min \left\{\left[(1+\epsilon) a_{n}\right], n\right\}$ that,

$$
P\left(\sum_{j=1}^{I_{n}} h_{1}\left(X_{j}\right) \geq-\epsilon \hat{b}_{n}\right) \geq 1-\frac{I_{n} \zeta_{1}^{2}}{\left(\epsilon \hat{b}_{n}\right)^{2}} \rightarrow 1 \quad \text { as } n \rightarrow \infty .
$$

Hence Corollary ?? implies that

$$
\limsup _{n \rightarrow \infty} \frac{\hat{W}_{n}\left(a_{n}\right)}{\hat{b}_{n}}=\limsup _{n \rightarrow \infty} \frac{\hat{D}_{n}\left(a_{n}\right)}{\hat{b}_{n}}=\limsup _{n \rightarrow \infty} \frac{\hat{T}_{n}\left(a_{n}\right)}{\hat{b}_{n}}=m \gamma_{h_{1}}(1 / c) \quad \text { a.s. }
$$

If in addition the sequence $a_{n}$ satisfy the relation $\log \log n=o\left(\log \frac{n}{a_{n}}\right)$, then

$$
\lim _{n \rightarrow \infty} \frac{\hat{W}_{n}\left(a_{n}\right)}{\hat{b}_{n}}=\lim _{n \rightarrow \infty} \frac{\hat{D}_{n}\left(a_{n}\right)}{\hat{b}_{n}}=m \gamma_{h_{1}}(1 / c) \quad \text { a.s. }
$$

which may be called Csörgö- Révéz law for increments of nondegenerate U-statistics.

- Note that if $a_{n}=n$, then from (??) of Theorem ?? related to Corollary ?? we obtain the law of the iterated logarithm for nondegenerate U-statistics, i.e.,

$$
\limsup _{n \rightarrow \infty} \frac{n\left(U_{n}(h)-\vartheta\right)}{\sqrt{2 n m^{2} \zeta_{1}^{2} \log \log n}}=\limsup _{n \rightarrow \infty} \max _{m \leq k \leq n} \frac{k\left(U_{k}(h)-\vartheta\right)}{\sqrt{2 n m^{2} \zeta_{1}^{2} \log \log n}}=1 \text { a.s. }
$$

- If $a_{n}$ satisfy the condition $\frac{\log \left(n / a_{n}\right)}{\log \log n} \rightarrow c \geq 0$, this occur for example when $a_{n}=$ $n /(\log n)^{c}$, then

$$
\begin{aligned}
\hat{b}_{n} & =\frac{n}{(\log n)^{c}} \gamma_{h_{1}}\left(\frac{(\log n)^{c}}{n}\left(\log \left(\frac{n(\log n)^{c}}{n}\right)+\log \log n\right)\right) \\
& =\frac{n}{(\log n)^{c}} \gamma_{h_{1}}\left(\frac{(\log n)^{c}(c+1) \log \log n}{n}\right) \\
& \sim \sqrt{2 \zeta_{1}^{2}(c+1) n \log \log n .}
\end{aligned}
$$

Thus from (??) of Theorem ?? related to Corollary ?? we get the following form of the law of iterated logarithm for increments:

$$
\limsup _{n \rightarrow \infty} \frac{\hat{W}_{n}\left(a_{n}\right)}{\sqrt{2 n m^{2} \log \log n}}=\limsup _{n \rightarrow \infty} \frac{\hat{D}_{n}\left(a_{n}\right)}{\sqrt{2 n m^{2} \log \log n}}=\zeta_{1} \sqrt{1+c} .
$$

- Finally consider the increments given by Mason, i.e. assume that $a_{n}=o(\log n)$. It is already seen that the condition $(i)$ of Theorem ?? related to Corollary ?? is 
satisfied. The sequence $\hat{b}_{n}$ take the form, $\hat{b}_{n}=a_{n} \gamma_{h_{1}}\left(\frac{\log n}{a_{n}}\right)$. Since $\log \log n=o\left(\frac{\log n}{a_{n}}\right)$, it follows from condition (??) of Theorem ?? related to Corollary ?? that

$$
\lim _{n \rightarrow \infty} \frac{\hat{W}_{n}\left(a_{n}\right)}{a_{n} m \gamma_{h_{1}}\left(\frac{\log n}{a_{n}}\right)}=\lim _{n \rightarrow \infty} \frac{\hat{D}_{n}\left(a_{n}\right)}{a_{n} m \gamma_{h_{1}}\left(\frac{\log n}{a_{n}}\right)}=1 \quad \text { a.s. }
$$

provided the series in Proposition ?? converges. This is a part of the extended Erdös-Rényi law for U-statistics, which generalize the extended Erdös-Rényi law for sums of i.i.d. random variables obtained by Mason [?].

So far the a.s. asymptotic behavior of the statistics (??)-(??) normalized by $\hat{b}_{n}$ is obtained up to the growth rate of the sequence $a_{n}$. For example, suppose that the distribution function of $h_{1}\left(X_{1}\right)$ is a normal with mean zero and variance $\zeta_{1}^{2}<\infty$. On one hand, if the sequence $a_{n}$ does not grows at all, i.e. $a_{n}=\mathrm{c}$ for a positive number $c$, then may be the series in Proposition ?? fail to converge in general. In this case, we have that

$$
\hat{b}_{n}=a_{n} \gamma_{h_{1}}\left(\frac{\log n}{a_{n}}\right) \sim \sqrt{2 c \zeta_{1}^{2} \log n} .
$$

Hence, the series in Proposition ?? does not converges, and therefore Corollary ?? is not applied. On the other hand, if $a_{n}=\log \log n$, then

$$
\hat{b}_{n}^{2} \sim 2 \zeta_{1}^{2}(\log \log n)^{2}(\log n) .
$$

Hence, the series in Proposition ?? converge and Corollary ?? implies that,

$$
\lim _{n \rightarrow \infty} \frac{\hat{W}_{n}(\log \log n)}{\sqrt{2 m^{2} \zeta_{1}^{2}(\log \log n)^{2} \log n}}=\lim _{n \rightarrow \infty} \frac{\hat{D}_{n}(\log \log n)}{\sqrt{2 m^{2} \zeta_{1}^{2}(\log \log n)^{2} \log n}}=1 \quad \text { a.s. },
$$

This is in fact, what we means by the word partly.

In many situations the moment generating function of $h_{1}\left(X_{1}\right)$ does not exists, but one sided power moments exists, then based on Proposition ??, we generalize Theorem ?? in the following

Corollary 3.4.2. Consider a nondegenerate U-statistics $U_{n}(h)$ based on a kernel $h$ of degree $m$ with $E h^{2}\left(X_{1}, \ldots, X_{m}\right)<\infty$. Furthermore suppose that one of the following two conditions is satisfied:

1. $\int_{0}^{\infty} e^{t h_{1}^{b}(x)} d F(x)<\infty$, for some $t>0$ and $0<b \leq 1$, and $\frac{a_{n}}{(\log n)^{\frac{2}{b}-1}} \rightarrow \infty$ as $n \rightarrow \infty$.

2. $\int_{0}^{\infty} h_{1}^{p}(x) d F(x)<\infty$, for some $p>2, \quad \log n \int_{-\infty}^{-n} h_{1}^{2}(x) d F(x) \rightarrow 0$, and $a_{n}>$ $\frac{c n^{2 / p}}{\log n}>0$ for some $c>0$ and all large $n$. 
Then we have

$$
\limsup _{n \rightarrow \infty} \frac{\hat{D}_{n}\left(a_{n}\right)}{\hat{b}_{n}}=\limsup _{n \rightarrow \infty} \frac{\hat{W}_{n}\left(a_{n}\right)}{\hat{b}_{n}}=\limsup _{n \rightarrow \infty} \frac{\hat{R}_{n}\left(a_{n}\right)}{\hat{b}_{n}}=\limsup _{n \rightarrow \infty} \frac{\hat{T}_{n}\left(a_{n}\right)}{\hat{b}_{n}}=1 \quad \text { a.s. }
$$

If in addition $\lim _{n \rightarrow \infty} \frac{\log \left(\frac{n}{a_{n}}\right)}{\log \log n}=\infty$ is satisfied, then

$$
\lim _{n \rightarrow \infty} \frac{\hat{D}_{n}\left(a_{n}\right)}{\hat{b}_{n}}=\lim _{n \rightarrow \infty} \frac{\hat{W}_{n}\left(a_{n}\right)}{\hat{b}_{n}}=1 \quad \text { a.s. },
$$

where $\hat{b}_{n}$ defined in (??).

Proof: In this case the series in Proposition ?? converge and the proof follows then by Theorem ?? and proposition ?? via the representation (??).

Remark 3.4.1. Let the assumptions of Corollary ?? are satisfied for both $h_{1}\left(X_{i}\right)$ and $-h_{1}\left(X_{i}\right)$. Then the assertion of Corollary ?? holds with -1 instead of 1 , if we replace limsup by liminf and sup by inf in the definition of statistics given by (??) - (??).

\subsubsection{Examples}

In the following example we give a nontrivial kernel $h$, which indicate that the statistics introduced by (??) - (??) generalize the statistics given by Erdös-Rényi, Shepp and Csörgö-Révéz.

Example 3.4.1. Consider a U-statistics $U_{n}(h)$ based on a kernel $h$ given by $h\left(x_{1}, x_{2}\right):=$ $\frac{x_{1}+x_{2}}{2}$ and w.l.o.g assume that $\vartheta:=E h\left(X_{1}, X_{2}\right)=0$. Then

$$
\begin{aligned}
\hat{D}_{n}\left(a_{n}\right) & :=\max _{2 \leq k \leq n-a_{n}}\left\{\left(k+a_{n}\right)\left(U_{k+a_{n}}(h)-\vartheta\right)-k\left(U_{k}(h)-\vartheta\right)\right\} \\
& =\max _{2 \leq k \leq n-a_{n}}\left\{\frac{k+a_{n}}{\left(\begin{array}{c}
k+a_{n} \\
2
\end{array}\right)} \sum_{1 \leq i<j \leq k+a_{n}}\left(X_{i}+X_{j}\right) / 2-\frac{k}{\left(\begin{array}{c}
k \\
2
\end{array}\right)} \sum_{1 \leq i<j \leq k}\left(X_{i}+X_{j}\right) / 2\right\} \\
& =\max _{2 \leq k \leq n-a_{n}}\left(S_{k+a_{n}}-S_{k}\right) .
\end{aligned}
$$

Which is the same statistic given by Erdös and Rényi [?].

Example 3.4.2. Let $\left\{X_{i}: i \geq 1\right\}$ be i.i.d. standard normally distributed random variables. Let $h: \mathbb{R}^{2} \rightarrow \mathbb{R}$ given by $h\left(x_{1}, x_{2}\right):=\frac{x_{1}+x_{2}}{2}$, and set $a_{n}=[c \log n]$ for positive 
number $c$. Then $h$ is nondegenerate and the assumptions of Corollary ?? are satisfied. Hence

$$
\frac{\hat{D}_{n}([c \log n])}{[c \log n]} \rightarrow 2 \gamma_{h_{1}}(1 / c) \text { a.s. } \forall c>0 .
$$

Let us now find $2 \gamma_{h_{1}}(1 / c)$ and compare it with the limit found by Erdös-Rényi. In this example we have $h_{1}\left(x_{1}\right)=\frac{x_{1}}{2}$, therefore $\varphi_{h_{1}}(t):=E\left(e^{t h_{1}}\right)=e^{t^{2} / 8}, c_{0}=0$ and $\xi_{h_{1}}(z)=$ $\sup _{t \in \mathbb{R}}\left\{z t-\log \varphi_{h_{1}}(t): \varphi_{h_{1}}(t)<\infty\right\}=2 z^{2}$, hence

$$
2 \gamma_{h_{1}}(1 / c)=2 \sup \left\{z: \xi_{h_{1}}(z) \leq 1 / c\right\}=2 \sup \left\{z: 2 z^{2} \leq 1 / c\right\}=2 \sqrt{1 / 2 c}=\sqrt{2 / c},
$$

which is the same limit given by Erdös and Rényi law for a sequence of i.i.d. $N(0,1)$ random variables.

Example 3.4.3. Let $\left\{X_{i}: i \geq 1\right\}$, be i.i.d. $N(0,1)$ random variables. For the $U$-statistics corresponding to the sample variance, one used the kernel $h$ given by $h\left(x_{1}, x_{2}\right):=\frac{(x-y)^{2}}{2}$. Then according to Corollary ?? with $a_{n}=[c \log n]$, we obtain

$$
\frac{\hat{D}_{n}([c \log n])}{[c \log n]} \rightarrow 2 \gamma_{h_{1}}(1 / c) \quad \text { a.s. } \forall c>c_{0} .
$$

Here we have $h_{1}(x)=\frac{x^{2}-1}{2}$, hence $\varphi_{h_{1}}(t)=\frac{e^{-t / 2}}{\sqrt{1-t}}, t<1$ and $m_{h_{1}}(t)=\frac{t}{2(1-t)}, A=$ $\infty, c_{0}=0$ and $\xi_{h_{1}}(z)=\sup _{t<1}\left\{z t-\log \left(\frac{e^{-t / 2}}{\sqrt{1-t}}\right)\right\}=\frac{z(2 z+1)}{2 z-1}+\frac{1}{2} \log (1-2 z)$. and therefore $2 \gamma_{h_{1}}(1 / c)=2 \sup \left\{z: \xi_{h_{1}}(z) \leq 1 / c\right\}$.

Example 3.4.4. Let $\left\{X_{i}: i \geq 1\right\}$, be i.i.d. $N\left(\mu, \sigma^{2}\right)$ random variables. Let $h: \mathbb{R}^{2} \rightarrow \mathbb{R}$ given by $h\left(x_{1}, x_{2}\right):=x_{1} x_{2}$, and let $E X_{1}=\mu \neq 0$. Then according to Corollary ?? we have,

$$
\frac{\hat{D}_{n}([c \log n])}{[c \log n]} \rightarrow 2 \gamma_{h_{1}}(1 / c) \quad \text { a.s. } \forall c>c_{0} .
$$

Here we have $\phi_{h_{1}}(t)=e^{\mu^{2} \sigma^{2} t^{2} / 2}$ and

$\xi_{h_{1}}(z)=\sup _{t \in \mathbb{R}}\left\{z t-\log \varphi_{h_{1}}(t): \varphi_{h_{1}}(t)<\infty\right\}=z^{2} / 2 \mu^{2} \sigma^{2}$. Therefore, $\gamma_{h_{1}}(1 / c)=\sup \left\{z: \xi_{h_{1}}(z) \leq 1 / c\right\}=\mu \sigma(2 / c)^{1 / 2}$. Hence,

$$
\max _{2 \leq k \leq n-a_{n}}\left\{\frac{2}{k+a_{n}-1} \sum_{2 \leq i<j \leq k+a_{n}}\left(X_{i} X_{j}-\mu^{2}\right)-\frac{2}{k-1} \sum_{2 \leq i<j \leq k}\left(X_{i} X_{j}-\mu^{2}\right)\right\}
$$

converge to $2 \mu \sigma(2 / c)^{1 / 2}$ a.s. for all $c>c_{0}$ as $n \rightarrow \infty$. 


\section{Chapter 4}

\section{Large Deviations for U-Statistics}

To describe the a.s. asymptotic behavior of the statistics $D_{n}\left(r_{n}\right)$ given by (??) and other functional of type $D_{n}\left(r_{n}\right)$, we need certain theory. However Erdös and Rényi based their proof on Bahadur-Rao [?] which is the refinement of Chernoff [?] large deviation theorem. A similar theorem like Bahadur-Rao is not available for U-statistics. But Csörgö and Révéz [?] proved that Bahadur-Rao large deviation theorem is not necessary for their proof. In fact, Csörgö and Révéz proved that Chernoff theorem can be used to achieve the Erdös-Rényi and Shepp laws. Their approach motives us to extend Erdös-Rényi and Shepp laws for U-statistics. Moreover the proof of all results in the first three section of Chapter ?? share the use of exponential properties of certain large deviation probabilities.

In fact large deviation theory is a part of probability theory that deals with the description of events where a random quantity deviates from its mean by more than a normal amount, for example beyond what is described by central limit theorem.

Therefore we state some known results from large deviations theory relevant to our purpose and derive from them exponential inequalities, which we need to describe the a.s. asymptotic behavior of the statistics $D_{n}\left(r_{n}\right)$ given by (??) in the next chapter. In the first section we proof new forms of large deviation results for a nondegenerate U-statistics. In the last section we derive a few results related to large deviation theory for arbitrary U-statistics.

\subsection{Moderate Large Deviation Theorem for U-Statistics}

Consider a U-statistics $U_{n}(h)$ as defined in (??). If $U_{n}(h)$ is nondegenerate, then by the theory of U-statistics under some conditions on moments of the kernel $h$, the central limit theorem applies. More precisely, suppose $E h\left(X_{1}, \ldots, X_{m}\right)=0$ and $0<\zeta_{1}^{2}:=$ 
$\operatorname{Var}\left(\tilde{h}\left(X_{1}\right)\right)<\infty$. Then under finite second moments assumption on $h$, we have

$$
P\left(\left(m \zeta_{1}\right)^{-1} n^{1 / 2} U_{n}(h)>x\right) \rightarrow(1-\Phi(x)),
$$

uniformly in $x$, as $n \rightarrow \infty$. Usually on a moderate large deviation, we are interested in the relative error of

$$
P\left(\left(m \zeta_{1}\right)^{-1} n^{1 / 2} U_{n}(h)>x_{n}\right)=\left(1-\Phi\left(x_{n}\right)\right)(1+o(1)),
$$

as $x_{n}$ tends to $\infty$ together with $n$. Malevich and Abdalimov [?] proved that the relation (??) holds for $x_{n}=O(\sqrt{\log n})$ under the condition that $E\left|h\left(X_{1}, \ldots, X_{m}\right)\right|^{k} \leq C^{k} k^{\gamma k}$, $k=1,2, \ldots$, for some constants $C>0$ and $\gamma \geq 0$. For more details on this problem, we refer the interested reader to the works done by, Aleskevicienè, [?, ?], Borovskikh and Weber [?, ?], Keener et. al. [?], Vandemaele [?, ?]. Among all results of this type, we will consider the works done by Aleskeviciene, [?], and Borovskikh and Weber [?,?]. The following theorem due to Aleskeviciene [?].

Theorem 4.1.1. Consider a nondegenerate U-statistics $U_{n}(h)$ with $E h\left(X_{1}, \ldots, X_{m}\right)=0$ and $0<\zeta_{1}^{2}<\infty$. If there exist a constants $C>0$ and $\lambda \geq 2$ such that, for all $k=2,3, \ldots$,

$$
E\left|h\left(X_{1}, \ldots, X_{m}\right)\right|^{k} \leq C^{k}(k !)^{\lambda} .
$$

Then as $n \rightarrow \infty$,

$$
\begin{aligned}
& P\left(\frac{\sqrt{n}}{m \zeta_{1}} U_{n}(h) \geq x\right)=(1-\Phi(x))\left(1+O\left(\frac{1+x^{3}+x \log n}{\sqrt{n}}\right)\right), \\
& P\left(\frac{\sqrt{n}}{m \zeta_{1}} U_{n}(h)<-x\right)=\Phi(-x)\left(1+O\left(\frac{1+x^{3}+x \log n}{\sqrt{n}}\right)\right)
\end{aligned}
$$

for all $x$ from the range $0 \leq x \leq c_{\lambda} n^{1 /(4 \lambda-2)}$, where

$$
c_{\lambda}=\min \left\{\frac{1}{2}, \frac{\zeta_{1}}{3 m^{4} 3 C(2.7)^{\lambda}},\left(3 C^{1 / \lambda}\right)^{-\lambda /(2 \lambda-1)}\right\} .
$$

Remark 4.1.1. The condition (??) is equivalent to the condition that there exists a constant $t_{0}>0$ such that $E e^{t\left|h\left(X_{1}, \ldots, X_{m}\right)\right|^{1 / \lambda}}<\infty$ for $t \in\left[-t_{0}, t_{0}\right]$. In particular if $\lambda=2$, then the estimates in (??) holds for all $x$ from the range $0 \leq x \leq c_{2} n^{1 / 6}$ with $c_{2}=$ $\min \left\{1 / 2, \zeta_{1} / 3 m^{4} 3 C(2.7)^{2},(3 \sqrt{C})^{-2 / 3}\right\}$, provided that $E e^{t\left|h\left(X_{1}, \ldots, X_{m}\right)\right|^{1 / 2}}<\infty$.

Theorem ?? yields the following large deviation result, which play an important role in establishing the lower limit of the Csörgö -Révéz law for nondegenerate U-statistics in the next chapter (see, Lemma ??). 
Corollary 4.1.1. Consider a nondegenerate kernel $h$ of degree $m$ satisfying the assumptions of Theorem ??. Furthermore consider a nondecreasing sequence of natural numbers $a_{n} \leq n$ such that $\frac{a_{n}}{(\log n)^{2 \lambda-1}} \rightarrow \infty$. Set $\hat{b}_{n}:=\sqrt{2 m^{2} \zeta_{1}^{2} a_{n}\left(\log \left(n / a_{n}\right)+\log \log n\right)}$. Then for all $\delta>0$, $n$ large enough and any positive number $\beta$, we have

$$
P\left(a_{n} U_{a_{n}}(h)>\beta \hat{b}_{n}\right) \geq\left(\frac{a_{n}}{n \log n}\right)^{\beta^{2}(1+\delta)} .
$$

Proof: Set $d_{n}:=2 \log \left(\frac{n}{a_{n}} \log n\right)$, then an application of Theorem ?? with $x=\sqrt{d_{n}}$ gives

$$
\begin{aligned}
& P\left(a_{n} U_{a_{n}}(h)>\beta \hat{b}_{n}\right)=P\left(\frac{\sqrt{a_{n}} U_{a_{n}}(h)}{m \zeta_{1}}>\beta \sqrt{2\left(\log \left(n / a_{n}\right)+\log \log n\right)}\right) \\
& =\left(1-\Phi\left(\sqrt{d_{n}}\right)\left(1+O\left(\frac{1+d_{n}^{3}+d_{n} \log n}{\sqrt{a_{n}}}\right)\right) \quad \text { using }(? ?)\right. \\
& =\frac{e^{-\beta^{2} d_{n} / 2}}{\beta \sqrt{2 \pi d_{n}}}(1+o(1)) \\
& \geq \frac{1}{2 \sqrt{4 \pi \beta^{2} \log \left(\left(n / a_{n}\right) \log n\right)}} e^{-\beta^{2} \log \left(\left(n / a_{n}\right) \log n\right)} \quad \text { for } n \text { large enough } \\
& \geq\left(\frac{n}{a_{n}} \log n\right)^{-\delta \beta^{2}}\left(\frac{n}{a_{n}} \log n\right)^{-\beta^{2}} \quad(*) \\
& =\left(\frac{a_{n}}{n \log n}\right)^{\beta^{2}(1+\delta)},
\end{aligned}
$$

where we use the fact that $1-\Phi(x)=\frac{e^{\frac{-x^{2}}{2}}}{x \sqrt{2 \pi}}(1+o(1))$ as $x \rightarrow \infty$ and in $(*)$ we take $\mathrm{n}$ large enough such that $\left(16 \pi \beta^{2} \log \left(\frac{n}{a_{n}} \log n\right)\right)^{-1 / 2} \geq\left(\frac{n}{a_{n}} \log n\right)^{-\delta \beta^{2}}$ holds.

Keener, Robinson, and Weber [?] proposed a contraction technique for establishing Cramer-type large deviation theorems for U-statistics. Their approach is a natural generalization of the classical methods of Cramer. Instead of stating their results, we state its generalization given by Borovskikh and Weber [?]. For this purpose, some notations are needed. Define

$$
c(x):=\left(E h^{2}\left(X_{1}, \ldots, X_{m-1}, x\right)\right)^{1 / 2}, x \in E \quad \text { and }\|c\|:=\left(E c^{2}(x)\right)^{1 / 2} .
$$

For some $a>0$, denote $d:=\left(E e^{a c(X)}\right)^{1 / 2}$ and $\lambda=\left(\frac{152 m d\left(1+d^{2}\right)}{a \zeta_{1}}\right)\left(1+\frac{8 d^{2}\|c\|}{a \zeta_{1}^{2}}\right)$.

Finally $\log _{l} n:=\log \left(\log _{l-1} n\right)$ with $\log _{0} n=n$. 
Theorem 4.1.2. Consider a U-statistics of the form

$$
U_{n}:=\frac{n}{m} U_{n}(h)=\frac{(m-1) \ldots 2.1}{(n-1) \ldots(n-m+1)} \sum_{1 \leq i_{1}<\ldots<i_{m} \leq n} h\left(X_{i_{1}}, \ldots, X_{i_{m}}\right) .
$$

Assume that $U_{n}(h)$ is a nondegenerate $U$-statistics. Further assume that $h$ satisfies the Cramér condition, i.e., $\phi(t):=E e^{a\left|h\left(X_{1}, \ldots, X_{m}\right)\right|}<\infty$ for some $a>0$. Then for $x=$ $O\left(\frac{\sqrt{n}}{\log _{l} n}\right)$, where $l \geq 1$ is any fixed integer, we have,

$$
\frac{P\left(U_{n}>x \zeta_{1} \sqrt{n}\right)}{1-\Phi(x)}=\exp \left\{\frac{x^{3}}{\sqrt{n}} \lambda_{m}\left(\frac{x}{\sqrt{n}}\right)\right\}\left(1+O\left(\frac{1+x}{\sqrt{n}}\right)\right.
$$

with the Cramér series

$$
\lambda_{m}(u)=\sum_{k=0}^{\infty} \lambda_{m k} u^{k}
$$

where $\left|\lambda_{m k}\right| \leq \lambda^{k+3}, \quad k=0,1, \ldots$, and the power series in (??) converge for $|u|<\lambda^{-1}$. Furthermore, if $E e^{a_{0} h^{2}\left(X_{1}, \ldots, X_{m}\right)}<\infty$ then (??) is also satisfied in the region $O\left(\frac{\sqrt{n}}{\log _{l} n}\right) \leq x \leq o(\sqrt{n})$ for some constant $a_{0}>0$.

The next theorem is also due to Borovskikh and Weber [?], which handle the product kernel.

Theorem 4.1.3. Consider a U-statistics $U_{n}(h)$. Suppose that the kernel $h$ is given by $h\left(x_{1}, \ldots, x_{m}\right)=\prod_{i=1}^{m} x_{i}$. Assume that $E X_{1} \neq 0,0<\sigma^{2}=\operatorname{Var}\left(X_{1}\right)<\infty$, and $E e^{a\left|X_{1}\right|}<\infty$ for some $a>0$ are satisfied. Then for $x=o(\sqrt{n})$ and any fixed integer $m \geq 1$,

$$
\frac{P\left(U_{n}(h)-\mu^{m}>\frac{x}{\sqrt{n}} m \sigma|\mu|^{m-1}\right)}{1-\Phi(x)}=\exp \left\{\frac{x^{3}}{\sqrt{n}} \lambda_{m}\left(\frac{x}{\sqrt{n}}\right)\right\}\left(1+O\left(\frac{1+x}{\sqrt{n}}\right)\right.
$$

with the Cramér series $\lambda_{m}(u)=\sum_{k=0}^{\infty} \lambda_{m k} u^{k}$, which converges for $0 \leq u \leq \eta$, where $\eta$ is a small positive number.

The following two corollaries expresses the large deviation result in the form needed for establishing the extended Erdös-Rényi law for nondegenerate U-statistics of degree $m$ in the next chapter (see, Theorems ?? and ??, p....).

Corollary 4.1.2. Consider a nondegenerate U-statistics $U_{n}$ as defined in formula (??). Suppose that the kernel $h$ satisfies the assumptions of Theorem ??. Then for $0<\alpha<1$, 
$\beta>0$, and $n$ large enough

$$
P\left(U_{n}>\zeta_{1} \beta n^{\frac{\alpha+1}{2}}\right)=\frac{e^{\frac{-\beta^{2} n^{\alpha}}{2}\left(1-\beta n^{\frac{\alpha-1}{2}} \lambda_{m}\left(\beta n^{\frac{\alpha-1}{2}}\right)\right)}}{\sqrt{2 \pi \beta^{2} n^{\alpha}}}(1+o(1))
$$

Proof: Using the fact that $1-\Phi(x)=\frac{e^{\frac{-x^{2}}{2}}}{x \sqrt{2 \pi}}(1+o(1))$ as $x \rightarrow \infty$ and an application of Theorem ?? with $x=\beta n^{\alpha / 2}$, we obtain

$$
\begin{aligned}
P\left(U_{n}>\zeta_{1} \beta n^{\frac{\alpha+1}{2}}\right) & =P\left(U_{n}>\beta n^{\frac{\alpha}{2}} \zeta_{1} \sqrt{n}\right) \\
& =\left(1-\Phi\left(\beta n^{\frac{\alpha}{2}}\right)\right) e^{\beta^{3} n^{\frac{3 \alpha-1}{2}} \lambda_{m}\left(\beta n^{\frac{\alpha-1}{2}}\right)}\left(1+O\left(n^{\frac{\alpha-1}{2}}\right)\right) \\
& =\frac{e^{\frac{-\beta^{2} n^{\alpha}}{2}} e^{\beta^{3} n^{\frac{3 \alpha-1}{2}} \lambda_{m}\left(\beta n^{\frac{\alpha-1}{2}}\right)}}{\sqrt{2 \pi \beta^{2} n^{\alpha}}}(1+o(1)) \\
& =\frac{e^{\frac{-\beta^{2} n^{\alpha}}{2}\left(1-\beta n^{\frac{\alpha-1}{2}} \lambda_{m}\left(\beta n^{\frac{\alpha-1}{2}}\right)\right)}}{\sqrt{2 \pi \beta^{2} n^{\alpha}}}(1+o(1)) .
\end{aligned}
$$

Corollary 4.1.3. Let the assumptions of Theorem ?? are satisfied. Then for all $\beta>0$ and all sufficiently large $n$, there exists a sequence of numbers $\eta_{n}:=\eta_{n}\left(\lambda_{m}, \phi\right)$ depending on $\lambda_{m}$ and $\phi(t)$ such that $\eta_{n} \rightarrow 0$ as $n \rightarrow \infty$, and the following estimate for $0<\alpha<1$ holds,

$$
\frac{e^{\frac{-\beta^{2} n^{\alpha}}{2}\left(1+\left|\eta_{n}\right|\right)}}{2 \sqrt{2 \pi \beta^{2} n^{\alpha}}} \leq P\left(U_{n}>\zeta_{1} \beta n^{\frac{\alpha+1}{2}}\right) \leq \frac{3 e^{\frac{-\beta^{2} n^{\alpha}}{2}\left(1-\left|\eta_{n}\right|\right)}}{2 \sqrt{2 \pi \beta^{2} n^{\alpha}}}
$$

Proof: The proof is an easy consequence of Corollary ?? by noting that the Cramér series $\lambda_{m}(u)$ converges for all sufficiently small $u$. Since $0<\alpha<1$, the sequence $n^{\frac{\alpha-1}{2}}$ converges to 0 as $n \rightarrow \infty$, we can take $\eta_{n}=\beta n^{\frac{\alpha-1}{2}} \lambda_{m}\left(\beta n^{\frac{\alpha-1}{2}}\right)$ and choose $n$ large enough such that $1 / 2<1+o(1)<3 / 2$.

\subsection{Large Deviation for arbitrary U-Statistics}

The problem treated in this section is quite different and more difficult from the previous one. It is of interest to determine the limit

$$
\lim _{n \rightarrow \infty} \frac{1}{n} \log P\left(U_{n}(h)>\beta\right)=-g(\beta),
$$


for any number $\beta>E h\left(X_{1}, \ldots, X_{m}\right)$, and the limit function $g(\beta)$ is in a perfect situation continuous. To solve this problem, one of the techniques is like this: one writes a $\mathrm{V}$ statistics $V_{n}(h)$ as defined in (??) in terms of empirical measure then applies Sanov's theorem or its generalization, and finally uses the contraction principle if the limit is continuous. More details on this problem is found in Eichelsbacher and Löwe [?, ?].

Remark 4.2.1. It has been already known that large deviation theorems of this type for $V_{n}(h)$ is the same as $U_{n}(h)$ (see Eichelsbacher and Löwe [?, ?]).

\subsubsection{Some Inequalities for arbitrary U-Statistics}

In the sequel, we will say that the kernel $h\left(X_{1}, \ldots, X_{m}\right)$ satisfies the condition (H2), if it satisfies the following conditions

1. $0 \leq E h\left(X_{1}, \ldots, X_{m}\right)<\infty$,

2. For all $x \in \mathbb{R}$, let $P\left(h\left(X_{1}, \ldots, X_{m}\right)=x\right)<1$,

3. $t_{0}:=\sup \left\{t: \phi(t)=E e^{t h\left(X_{1}, \ldots, X_{m}\right)}<\infty\right\}>0$.

If the kernel $h$ satisfying the condition (H2), then for $t<t_{0}$, we define a collection of basic functions and constants

$$
\varphi_{h}(t), m_{h}(t), \sigma_{h}^{2}(t), f_{h}(t), \omega_{h}, \xi_{h}(\alpha) \text { and } \gamma_{h}(x),
$$

by (??) - (??) with $X$ replaced by $h\left(X_{1}, \ldots, X_{m}\right)$.

Their properties may be derived in a similar way as given in Lemma ??. We will see that this collection of the basic functions and constants play an essential role in describing the asymptotic limiting behavior of statistics introduced in the next chapter.

Lemma 4.2.1. Let $E e^{t h\left(X_{1}, \ldots, X_{m}\right)}<\infty$ for some $t>0$ and a positive integer $m<n$. Then for all $x \geq 0$, we have

$$
\limsup _{n \rightarrow \infty} \frac{1}{n} \log P\left(U_{n}(h) \geq x\right) \leq \frac{-1}{m} \xi_{h}(x) .
$$




\section{Proof.}

$$
\begin{aligned}
P\left(U_{n}(h) \geq x\right) & \left.=P\left(\left(\begin{array}{c}
n \\
m
\end{array}\right)^{-1} \sum_{1 \leq i_{1}<\ldots<i_{m} \leq n}[n / m] h\left(X_{i_{1}}, \ldots, X_{i_{m}}\right)\right)>x[n / m]\right) \\
& \leq e^{-t x[n / m]} E e^{t U_{n}([n / m] h)} \\
& =e^{-t x[n / m]} E e^{\frac{1}{n !} \sum_{P e r} t[n / m] W\left(X_{i_{1}}, \ldots, X_{i_{n}}\right)} \\
& \leq e^{-t x[n / m]} \frac{1}{n !} \sum_{P e r} E e^{t[n / m] W\left(X_{i_{1}}, \ldots, X_{i_{n}}\right)} \\
& =e^{-t x[n / m]} E e^{t[n / m] \frac{1}{[n / m]} \sum_{p=1}^{[n / m]} h\left(X_{p m-m+1}, \ldots, X_{p m}\right)} \\
& =e^{-t x[n / m]}\left(E e^{t h\left(X_{1}, \ldots, X_{m}\right)}\right)^{[n / m]}
\end{aligned}
$$

where we used Hoeffeding representation given in formula (??) and the convexity property of the exponential function. Therefore we have,

$$
\begin{aligned}
P\left(U_{n}(h) \geq x\right) & \leq\left(\inf _{t} e^{-t x} E e^{t h\left(X_{1}, \ldots, X_{m}\right)}\right)^{[n / m]} \\
& =e^{\left[\frac{-n}{m}\right] \sup _{t}\left(t x-\log E e^{t h\left(X_{1}, \ldots, X_{m}\right)}\right)} .
\end{aligned}
$$

Hence, the lemma is proved by taking logarithm and lim sup as $n \rightarrow \infty$ for both side.

The following proposition play an important role in establishing the upper limit of the Csörgö -Révéz law for arbitrary U-statistics of degree $m$ in the next chapter (see, Lemma ??, p....).

Proposition 4.2.1. Let $E e^{t h\left(X_{1}, \ldots, X_{m}\right)}<\infty$ for some $t>0$ and a positive integer $m$. Furthermore, let $E h\left(X_{1}, \ldots, X_{m}\right)=0$ and $0<\zeta_{m}^{2}:=E h^{2}\left(X_{1}, \ldots, X_{m}\right)<\infty$. Set

$$
\tilde{b}_{n}=\sqrt{2 \beta^{2} m \zeta_{m}^{2} a_{n}\left(\log \left(n / a_{n}\right)+\log \log n\right)} .
$$

Then, for all $\beta>0, \delta>0$ and $a_{n}$ with $\frac{a_{n}}{\log n} \rightarrow \infty$, we have

$$
P\left(a_{n} U_{a_{n}}(h)>\beta \tilde{b}_{n}\right) \leq\left(\frac{a_{n}}{n \log n}\right)^{\beta^{2}(1-\delta)}
$$

Proof: By Lemma ?? we have

$$
\begin{aligned}
P\left(a_{n} U_{a_{n}}(h) \geq \beta \tilde{b}_{n}\right) & =P\left(U_{a_{n}}(h) \geq \sqrt{2 \beta^{2} m \zeta_{m}^{2}\left(\log \left(n / a_{n}\right)+\log \log n\right) / a_{n}}\right) \\
& \leq e^{\frac{-a_{n}}{m} \xi_{h}}\left(\sqrt{2 \beta^{2} m \zeta_{m}^{2} \log \left(\frac{n}{a_{n}} \log n\right) / a_{n}}\right)
\end{aligned}
$$


Since $\frac{a_{n}}{\log n} \rightarrow \infty$, then the sequence $\frac{2 \beta^{2} m \zeta_{m}^{2} \log \left(\frac{n}{a_{n}} \log n\right)}{a_{n}} \rightarrow 0$. Therefore, we want to find an asymptotic formula of $\xi_{h}(x)$. A second order expansion of the logarithm function yields

$$
\begin{aligned}
c(t) & :=\log E e^{t h\left(X_{1}, \ldots, X_{m}\right)} \\
& =c(0)+t c^{\prime}(0)+\frac{t^{2}}{2 !} c^{\prime \prime}(0)+O\left(t^{3}\right) \\
& =c(0)+t E h\left(X_{1}, \ldots, X_{m}\right)+\frac{t^{2}}{2 !} E h^{2}\left(X_{1}, \ldots, X_{m}\right)+O\left(t^{3}\right) \\
& =\frac{\zeta_{m}^{2}}{2} t^{2}+O\left(t^{3}\right) \quad(\text { as } t \rightarrow 0) .
\end{aligned}
$$

Hence, as $x$ approaches 0 , we obtain

$$
\xi_{h}(x):=\sup _{t}\{t x-c(t)\}=\frac{x^{2}}{2 \zeta_{m}^{2}}+O\left(x^{3}\right) .
$$

Therefore for $\mathrm{n}$ large enough we get,

$$
\begin{aligned}
& \frac{a_{n}}{m} \xi_{h}\left(\sqrt{2 \beta^{2} m \zeta_{m}^{2}\left(\log \left(n / a_{n}\right)+\log \log n\right) / a_{n}}\right)= \\
& =\frac{a_{n}}{m}\left(\frac{2 \beta^{2} m \zeta_{m}^{2}}{2 \zeta_{m}^{2} a_{n}} \log \left(\frac{n}{a_{n}} \log n\right)\right)+\frac{a_{n}}{m} O\left(\left(2 \beta^{2} m \zeta_{m}^{2} \log \left(\frac{n \log n}{a_{n}}\right)\right)^{3 / 2} / a_{n}^{3 / 2}\right) \\
& =\beta^{2} \log \left(\frac{n}{a_{n}} \log n\right)+O\left(\left(2 \beta^{2} \zeta_{m}^{2} \log \left(\frac{n \log n}{a_{n}}\right)\right)^{3 / 2} / \sqrt{a_{n}}\right) \\
& =\beta^{2} \log \left(\frac{n}{a_{n}} \log n\right)\left(1+O\left(\sqrt{\left(\log \left(n / a_{n}\right)+\log \log n\right) / a_{n}}\right)\right) \\
& \geq \beta^{2}(1-\delta) \log \left(\frac{n}{a_{n}} \log n\right) \quad \text { for all } \delta>0 \text { and } n \text { large enough. }
\end{aligned}
$$

We complete the proof by replacing the last inequality in (??).

The following proposition is essential in establishing the upper limit of the extended Erdös-Rényi law given by Mason [?], and Erdös-Rényi and Shepp laws for arbitrary Ustatistics of degree $m$ (see, Lemma ?? and Lemma ?? p....).

Proposition 4.2.2. For any number $\beta>0$ and integer $n \geq m$, we have

$$
P\left(U_{a_{n}}(h) \geq m \beta \gamma_{h}\left(\frac{\beta_{n}}{a_{n}}\right)\right) \leq e^{-\beta \beta_{n}} .
$$


Proof. This follows from an application of Lemma ?? with $x=m \beta \gamma_{h}\left(\beta_{n} / a_{n}\right)$, and using the fact that $\xi_{h}()$ is a convex function. Thus by the definition of convexity, we have $\xi_{h}(v) \leq \frac{w-v}{w-u} \xi_{h}(u)+\frac{v-u}{w-u} \xi_{h}(w)$, for $u<v<w$. By choosing $u=0, v=\gamma_{h}\left(\beta_{n} / a_{n}\right)$ and $w=m \beta \gamma_{h}\left(\beta_{n} / a_{n}\right)$ and noting that $\xi_{h}(0)=0$. These facts together imply that

$$
\begin{aligned}
P\left(U_{n}(h) \geq m \beta \gamma_{h}\left(\beta_{n} / a_{n}\right)\right) & \leq e^{\frac{-a_{n}}{m} \xi_{h}\left(m \beta \gamma_{h}\left(\beta_{n} / a_{n}\right)\right)} \\
& \leq e^{\frac{-a_{n}}{m} m \beta \xi_{h}\left(\gamma_{h}\left(\frac{\beta_{n}}{a_{n}}\right)\right)} \\
& \leq e^{-\beta \beta_{n}} .
\end{aligned}
$$

The last inequality follows by the definition of $\gamma_{h}()$ and by observing that $\xi_{h}\left(\gamma_{h}\left(\beta_{n} / a_{n}\right)\right) \geq \beta_{n} / a_{n}$.

Consider a U-statistics $U_{n}(h)$ based on a kernel $h$ of degree 2 . Then by using the Hdecomposition Theorem ??, and the expansion given in formula (??). We rewrite $U_{n}(h)$ as follows,

$$
\begin{aligned}
U_{n}(h) & =\vartheta+\frac{2}{n} \sum_{i=1}^{n} h_{1}\left(X_{i}\right)+\left(\begin{array}{l}
n \\
2
\end{array}\right)^{-1} \sum_{1 \leq i<j \leq n} h_{2}\left(X_{i}, X_{j}\right) \\
& =\frac{1}{n} \sum_{i=1}^{n}\left(2 h_{1}\left(X_{i}\right)+\vartheta\right)+\sum_{p=1}^{\infty}\left(\begin{array}{c}
n \\
2
\end{array}\right)^{-1} \sum_{1 \leq i<j \leq n} \nu_{p} \psi_{p}\left(X_{i}\right) \psi_{p}\left(X_{j}\right) \\
& =\frac{1}{n} \sum_{i=1}^{n}\left(2 h_{1}\left(X_{i}\right)+\vartheta\right)+\sum_{p=1}^{\infty} \nu_{p}\left(n^{-1} \sum_{i=1}^{n} \psi_{p}\left(X_{i}\right)\right)^{2}- \\
& -\sum_{p=1}^{\infty} \nu_{p} n^{-2} \sum_{i=1}^{n} \psi_{p}^{2}\left(X_{i}\right) .
\end{aligned}
$$

Theorem 4.2.1. (Arcones (1992))

Consider a U-statistics $U_{n}(h)$ based on a kernel $h$ of degree $m=2$, and assume that the kernel $h$ admits the representation as given in formula (??). Furthermore suppose that, for each $t>0$, the following condition holds

$$
E e^{t\left(\sum_{p=0}^{\infty}\left|\nu_{p}\right| \psi_{p}^{2}(X)\right)^{1 / 2}}<\infty
$$

where $\nu_{0}=1$ and $\psi_{0}=2 h_{1}\left(X_{i}\right)+\vartheta$. For $x \in l_{2}$, where

$$
l_{2}:=\left\{x=\left(x_{0}, x_{1}, \ldots\right): \sum_{p=0}^{\infty} x_{p}^{2}<\infty\right\},
$$


$r \in \mathbb{R}$ and $A \subset \mathbb{R}$ define

$$
\begin{aligned}
& \lambda_{1}(x):=\sup _{\xi \in l_{2}}\left\{\sum_{p=0}^{\infty} \xi_{p} x_{p}-\log E e^{\sum_{p=0}^{\infty} \xi_{p} \nu_{p} \psi_{p}(X)}\right\}, \quad \text { for } x \in l_{2}, \\
& \lambda(r):=\inf \left\{\lambda_{1}(x): x_{0}+\sum_{p=1}^{\infty} \operatorname{sign}\left(\nu_{p}\right) x_{p}^{2}=r\right\}, \quad \text { for } r \in \mathbb{R} \text {, and } \\
& \Lambda(A):=\inf \{\lambda(r): r \in A\} \text {. }
\end{aligned}
$$

Then for any closed set $F$ and any open set $G$ in $\mathbb{R}$ the following holds

$$
\begin{array}{r}
-\Lambda(F) \geq \limsup _{n \rightarrow \infty} \frac{1}{n} \log P\left(U_{n}(h) \in F\right) \quad \text { and } \\
-\Lambda(G) \leq \liminf _{n \rightarrow \infty} \frac{1}{n} \log P\left(U_{n}(h) \in G\right) .
\end{array}
$$

Corollary 4.2.1. Under the assumption of Theorem ??, for $\epsilon>0$ and $n$ large enough, we have

$$
e^{-n(\Lambda(A)+\epsilon)} \leq P\left(U_{n}(h) \geq \alpha\right) \leq e^{-n(\Lambda(\bar{A})-\epsilon)} .
$$

Proof: Set $A=\left(\alpha, \omega_{h}\right)$ and $\bar{A}=\left[\alpha, \omega_{h}\right]$, where $\omega_{h}:=e s s \sup h\left(X_{1}, \ldots, X_{m}\right)$. Then, the assertion follows immediately from Theorem ??.

Example 4.2.1. Let $\left\{X_{i}: i \geq 1\right\}$, be i.i.d. $N\left(0, \sigma^{2}\right)$ random variables. Suppose that the kernel $h$ given by $h(x, y)=x y$. Then $h_{1}\left(x_{1}\right)=E h\left(x_{1}, X_{2}\right)=x_{1} E X_{2}=0$ and $h_{2}\left(x_{1}, x_{2}\right)=x_{1} x_{2}$. Hence, $\nu_{0}=0, \nu_{1}=\sigma^{2}, \nu_{p}=\psi_{p}(x)=0$ for all $p \geq 2, \psi_{0}=0$, and $\psi_{1}(x)=x / \sigma$. Therefore, we write $h(x, y)$ as

$$
x y=\beta_{1} \psi_{1}(x) \psi_{1}(y)=\sigma^{2} \frac{x}{\sigma} \frac{y}{\sigma} .
$$

Let us calculate the functionals defined on Theorem ??.

$$
\begin{gathered}
\lambda_{1}(x)=\sup _{\xi \in l_{2}}\left\{\sum_{p=0}^{\infty} \xi_{p} x_{p}-\log E e^{\sigma^{2} \xi_{1} \frac{x_{1}}{\sigma}}\right\}=\sup _{\xi \in l_{2}}\left\{\sum_{p=0}^{\infty} \xi_{p} x_{p}-\frac{1}{2} \sigma^{2} \xi_{1}^{2}\right\}=\frac{x_{1}^{2}}{2 \sigma^{2}} . \\
\lambda(r)=\inf _{x \in l_{2}}\left\{\frac{x_{1}^{2}}{2 \sigma^{2}}: x_{0}+\sigma^{2} x_{1}^{2}=r\right\}=\frac{r}{4 \sigma^{2}} .
\end{gathered}
$$

Finally

$$
\Lambda(A)=\inf _{r \in\left(\alpha, \omega_{h}\right)} \lambda(r)=\frac{\alpha}{4 \sigma^{2}} .
$$




\section{Chapter 5}

\section{Strong Laws for Increments of U-Statistics}

This chapter contains strong laws for increments for U-statistics, which also generalize the strong laws for increments of partial sums given in the first three sections of Chapter ??. The proof heavily depend on the results explained in Chapter ??. For any nondecreasing sequence of natural numbers $m \leq r_{n} \leq n$ set

$$
U_{1+k}^{k+r_{n}}:=\sum_{1+k \leq i_{1}<\ldots<i_{m} \leq k+r_{n}} h\left(X_{i_{1}}, \ldots, X_{i_{m}}\right) .
$$

With the statistic

$$
D_{n}\left(r_{n}\right):=\max _{0 \leq k \leq n-r_{n}} U_{1+k}^{k+r_{n}}
$$

we define functionals or statistics of this type as follows:

$$
\begin{gathered}
T_{n}\left(r_{n}\right):=U_{1+n}^{n+r_{n}}, \\
W_{n}\left(r_{n}\right):=\max _{0 \leq k \leq n-r_{n}} \max _{m \leq j \leq r_{n}} U_{1+k}^{k+j} \text { and } \\
R_{n}\left(r_{n}\right):=U_{1+n-r_{n}}^{n} \text {, and } \tilde{R}_{n}\left(r_{n}\right):=\max _{m \leq k \leq r_{n}} U_{1+n}^{n+k} .
\end{gathered}
$$

First of all, let us look at some special cases of the statistics (??) - (??).

- Let $m=2$ and $h$ be given by $h(x, y)=(x+y)$, then the statistics (??) - (??) are 
the same as statistics (??) - (??) multiplied with $r_{n}-1$.

- Let $r_{n}=n$. Then

$$
D_{n}(n)=\left(\begin{array}{c}
n \\
m
\end{array}\right) U_{n}(h) \quad \text { and } \quad W_{n}(n)=\max _{m \leq j \leq n}\left(\begin{array}{c}
j \\
m
\end{array}\right) U_{j}(h) .
$$

- Let $r_{n}=m$. Then

$$
D_{n}(m)=W_{n}(m)=\max _{0 \leq k \leq n-2} h\left(X_{k+1}, X_{k+2}, \ldots, X_{k+m}\right) .
$$

Hence, the statistics given by (??) - (??), the theory of sums of U-statistics, and the extreme value theory for $\mathrm{m}$-dependent random variables are parts of the general theory of statistics (??) - (??).

\subsection{Erdös-Rényi and Shepp Laws for U-Statistics}

In this section, we describe the a.s. asymptotic behavior of the statistics $D_{n}\left(r_{n}\right)$ defined in (??) considering increments of length $[c \log n]$ with positive number $c$. Thus, we generalize the Erdös-Rényi law for sums of i.i.d. random variables Theorem ??. The following theorem holds for arbitrary kernel of degree $m=2$ satisfying some moment condition, no restriction on the degeneracy of $h$ is made. We use the notations from Theorem ??.

Theorem 5.1.1. (Erdös-Rényi Law for arbitrary U-Statistics with $m=2$ )

Consider a U-statistics $U_{n}(h)$ based on a kernel $h$ of degree $m=2$. Assume that $U_{n}(h)$ admits the representation (??). Furthermore suppose that, for each $t>0$,

$$
\phi(t):=E e^{t\left(\sum_{p=0}^{\infty}\left|\nu_{p}\right| \psi_{p}^{2}(X)\right)^{1 / 2}}<\infty
$$

holds. Let $\alpha \in\left(\vartheta, \omega_{h}\right)$ and set $B_{\alpha}:=\left[\alpha, \omega_{h}\right]$, where $\omega_{h}:=e s s \sup h\left(X_{1}, \ldots, X_{m}\right)$. For $a$ positive number $c>0$, set $r_{n}=[c \log n]$. Then the rate function $\Lambda\left(B_{\alpha}\right)$ exists and if it is in addition strictly increasing in $\alpha$. Then

$$
\lim _{n \rightarrow \infty} \frac{D_{n}\left(r_{n}\right)}{\left(\begin{array}{c}
r_{n} \\
2
\end{array}\right)}=\Gamma(c) \quad \text { a.s. }
$$

where $\Gamma(c):=\sup \left\{\alpha: \Lambda\left(B_{\alpha}\right) \leq 1 / c\right\}$. 
Proof. Let $\delta>0$ such that $(\Gamma(c)+\delta) \in\left(\vartheta, \omega_{h}\right)$, then

$$
\begin{aligned}
P\left\{D_{n}\left(r_{n}\right) \geq\left(\begin{array}{c}
r_{n} \\
2
\end{array}\right)(\Gamma(c)+\delta)\right\} & =P\left\{\max _{0 \leq k \leq n-r_{n}} U_{1+k}^{k+r_{n}} \geq\left(\begin{array}{c}
r_{n} \\
2
\end{array}\right)(\Gamma(c)+\delta)\right\} \\
& \leq \sum_{k=0}^{n-r_{n}} P\left\{U_{1+k}^{k+r_{n}} \geq\left(\begin{array}{c}
r_{n} \\
2
\end{array}\right)(\Gamma(c)+\delta)\right\} \\
& \leq n P\left(U_{r_{n}}(h) \geq \Gamma(c)+\delta\right) \\
& \leq n e^{-r_{n}\left(\Lambda\left(B_{\Gamma(c)+\delta}\right)-\epsilon\right)}
\end{aligned}
$$

where the last inequality follows by using Corollary ?? for $n$ sufficiently large. Making use of the increasing sequence of integers, $n_{l}:=\max \{n:[c \log n]=l\}$.

Then for $l$ sufficiently large

$$
\begin{aligned}
& P\left(D_{n_{l}}\left(r_{n_{l}}\right) \geq\left(\begin{array}{c}
r_{n_{l}} \\
2
\end{array}\right)(\Gamma(c)+\delta)\right) \leq n_{l} e^{-r_{n_{l}}\left(\Lambda\left(B_{\Gamma(c)+\delta}\right)-\epsilon\right)} \\
& \leq e^{\frac{l+1}{c}} e^{-l\left(\Lambda\left(B_{\Gamma(c)+\delta}\right)-\epsilon\right)} \leq e^{1 / c} e^{-l\left(\frac{-1}{c}+\frac{1}{c}+\delta_{0}\right)} \quad \text { for some } \delta_{0}:=\delta_{0}(c, \delta, \epsilon)>0 \\
& =e^{1 / c} e^{-l \delta_{0}} .
\end{aligned}
$$

In the above estimation first of all, we may increase $l$ to make $\epsilon$ small enough. Also note that by the definition of $\Gamma(c)$ and the strict monotony assumption on $\Lambda($.$) , there exists$ $\delta_{0}>0$ depending on $c, \delta$ and $\epsilon$ such that $\Lambda\left(B_{\Gamma(c)+\delta}\right)-\epsilon \geq \frac{1}{c}+\delta_{0}$. Therefore, we have by the integral test that,

$$
\sum_{l=M_{0}}^{\infty} P\left(D_{n_{l}}\left(r_{n_{l}}\right) \geq\left(\begin{array}{c}
r_{n_{l}} \\
2
\end{array}\right)(\Gamma(c)+\delta)\right)<\infty
$$

Notice that, for $n_{l-1}<n \leq n_{l}$, we have $[c \log n]=l$ and $D_{n}\left(r_{n}\right) \leq D_{n_{l}}\left(r_{n_{l}}\right)$. Thus, Borel-Cantelli lemma implies that

$$
\limsup _{n \rightarrow \infty}\left(\begin{array}{c}
r_{n} \\
2
\end{array}\right)^{-1} D_{n}\left(r_{n}\right) \leq \limsup _{l \rightarrow \infty}\left(\begin{array}{c}
r_{n_{l}} \\
2
\end{array}\right)^{-1} D_{n_{l}}\left(r_{n_{l}}\right) \leq \Gamma(c) \quad \text { a.s. },
$$

since $\delta>0$ can be taken arbitrary small.

To complete the proof of the theorem, we we need only to show

$$
\liminf _{n \rightarrow \infty}\left(\begin{array}{c}
r_{n} \\
2
\end{array}\right)^{-1} D_{n}\left(r_{n}\right) \geq \Gamma(c) \quad \text { a.s. }
$$


For $n$ sufficiently large and $\delta>0$, we have

$$
\begin{aligned}
& P\left(D_{n}\left(r_{n}\right) \leq\left(\begin{array}{c}
r_{n} \\
2
\end{array}\right)(\Gamma(c)-\delta)\right):=P\left(\max _{0 \leq k \leq n-r_{n}} U_{1+k}^{k+r_{n}} \leq(\Gamma(c)-\delta)\left(\begin{array}{c}
r_{n} \\
2
\end{array}\right)\right) \\
& \leq P\left(\max _{k=1, \ldots,\left[\frac{n}{r_{n}}\right]} U_{1+(k-1) r_{n}}^{k r_{n}} \leq(\Gamma(c)-\delta)\left(\begin{array}{c}
r_{n} \\
2
\end{array}\right)\right) \\
& =\left(1-P\left(U_{r_{n}}(h)>\Gamma(c)-\delta\right)\right)^{\left[\frac{n}{r_{n}}\right]} \quad \text { by independence of } U_{1+(k-1) r_{n}}^{k r_{n}} \\
& \leq\left(1-e^{-r_{n}\left(\Lambda\left(B_{\Gamma(c)-\delta}\right)+\epsilon\right)}\right)^{\left[\frac{n}{r_{n}}\right]} \text { by using Corollary ??. }
\end{aligned}
$$

Since $\Lambda\left(B_{(\Gamma(c)-\delta)}\right)<1 / c$, for any $\delta>0$ and $c>0$, there exists $\delta_{1}:=\delta_{1}(\epsilon, \delta, c)>0$ such that $\Lambda\left(B_{\Gamma(c)-\delta}\right)+\epsilon \leq \frac{1-\delta_{1}}{c}$. It follows that

$$
\begin{aligned}
P\left(D_{n}\left(r_{n}\right) \leq\left(\begin{array}{c}
r_{n} \\
2
\end{array}\right)(\Gamma(c)-\delta)\right) & \leq\left(1-e^{-r_{n}\left(\frac{1-\delta_{1}}{c}\right)}\right)^{\left[\frac{n}{r_{n}}\right]} \\
& \leq \exp \left\{-\left[n / r_{n}\right] e^{-(c \log n)\left(\frac{1-\delta_{1}}{c}\right)}\right\} \\
& \leq \exp \left\{-\frac{n}{r_{n}} n^{-c\left(\frac{1-\delta_{1}}{c}\right)}\right\} \\
& \leq e^{-\frac{n^{\delta_{1}}}{\log n}} \text { for some } \delta_{1}>0 .
\end{aligned}
$$

By integral test for all $n>N_{1}$, where $N_{1}$ large enough, we obtain

$$
\sum_{n=N_{1}}^{\infty} P\left(\left(\begin{array}{c}
r_{n} \\
2
\end{array}\right)^{-1} D_{n}\left(r_{n}\right) \leq \Gamma(c)-\delta\right)<\infty .
$$

Hence, Borel-Cantelli lemma implies that with probability one

$$
\liminf _{n \rightarrow \infty}\left(\begin{array}{c}
r_{n} \\
2
\end{array}\right)^{-1} D_{n}\left(r_{n}\right) \geq \Gamma(c)-\delta .
$$

Let $\delta \downarrow 0$ and obtain that

$$
\liminf _{n \rightarrow \infty}\left(\begin{array}{c}
r_{n} \\
2
\end{array}\right)^{-1} D_{n}\left(r_{n}\right) \geq \Gamma(c) \quad \text { a.s. }
$$

which complete the proof of the theorem. 


\subsubsection{Generalized Erdös-Rényi Law for U-Statistics}

We are going to prove the following theorem, which describe the a.s. asymptotic behavior of the statistics $D_{n}\left(r_{n}\right)$. The theorem cover only nondegenerate kernel of degree $m \geq 2$ and the increments are of length approximately $\left[(\log n)^{q}\right]$, for $q>1$. It generalize Theorem ?? given by Book [?].

Theorem 5.1.2. (Generalized Erdös-Rényi law for nondegenerate U-Statistics)

Consider a nondegenerate $U$-statistics $U_{n}(h)$ and without loss of generality assume that $E h\left(X_{1}, \ldots, X_{m}\right)=0$. For $\beta>0$ set $r_{n}=\left[\left(2 \beta^{-2} \log n\right)^{1 / \alpha}\right]$. Let the kernel $h$ satisfy the Cramér condition $E e^{t\left|h\left(X_{1}, \ldots, X_{m}\right)\right|}<\infty$ for some $t>0$. Then for each $0<\alpha<1$

$$
\lim _{n \rightarrow \infty}\left(\begin{array}{c}
r_{n} \\
m
\end{array}\right)^{-1} \frac{D_{n}\left(r_{n}\right)}{m \zeta_{1} r_{n}^{\frac{\alpha-1}{2}}}=\beta \quad \text { a.s. }
$$

Proof: Let $\epsilon>0$ and set $A_{n}:=\left\{D_{n}\left(r_{n}\right)>(1+\epsilon) \beta\left(\begin{array}{c}r_{n} \\ m\end{array}\right) r_{n}^{\frac{\alpha-1}{2}} m \zeta_{1}\right\}$. Then

$$
\begin{aligned}
P\left(A_{n}\right) & :=P\left\{\max _{0 \leq k \leq n-r_{n}} U_{1+k}^{k+r_{n}}>(1+\epsilon) \beta\left(\begin{array}{c}
r_{n} \\
m
\end{array}\right) r_{n}^{\frac{\alpha-1}{2}} m \zeta_{1}\right\} \\
& \leq \sum_{k=0}^{n-r_{n}} P\left\{\frac{r_{n}}{m}\left(\begin{array}{c}
r_{n} \\
m
\end{array}\right) U_{1+k}^{-1}>\beta(1+\epsilon) \zeta_{1} r_{n}^{\frac{\alpha+1}{2}}\right\} \\
& \leq n \cdot P\left\{U_{r_{n}}>\zeta_{1} \beta(1+\epsilon) r_{n}^{\frac{\alpha+1}{2}}\right\} \\
& \leq n \cdot \frac{3 e^{\frac{-\beta^{2}(1+\epsilon)^{2} r_{n}^{\alpha}}{2}\left(1-\left|\eta_{r_{n}}\right|\right)}}{2 \beta \sqrt{2 \pi r_{n}^{\alpha}}} \operatorname{using}(? ?) .
\end{aligned}
$$

Notice that $\left(\frac{r_{n}}{1+r_{n}}\right)^{\alpha} \rightarrow 1$. Thus for all $n \geq N_{1}$, we have $\left(\frac{r_{n}}{1+r_{n}}\right)^{\alpha}>1-\delta_{1}$. Also $\left(1-\left|\eta_{r_{n}}\right|\right) \rightarrow 1$, which implies that for all $n \geq N_{2}$, we have $1-\left|\eta_{r_{n}}\right|>1-\delta_{2}$. Hence, for all $n \geq N_{0}:=\max \left(N_{1}, N_{2}\right)$, the following estimates holds.

$$
\begin{aligned}
& \frac{\beta^{2}}{2}(1+\epsilon)^{2} r_{n}^{\alpha}\left(1-\left|\eta_{r_{n}}\right|\right)=\frac{1}{2} \beta^{2}(1+\epsilon)^{2}\left(\frac{r_{n}}{1+r_{n}}\right)^{\alpha}\left(1+r_{n}\right)^{\alpha}\left(1-\left|\eta_{r_{n}}\right|\right) \\
& \geq \frac{\beta^{2}}{2}(1+\epsilon)^{2}\left(\frac{r_{n}}{1+r_{n}}\right)^{\alpha}\left(1+\left(\frac{2 \log n}{\beta^{2}}\right)^{1 / \alpha}-1\right)^{\alpha}\left(1-\left|\eta_{r_{n}}\right|\right) \\
& \geq \frac{\beta^{2}}{2}(1+\epsilon)^{2}\left(1-\delta_{1}\right)\left(\frac{2 \log n}{\beta^{2}}\right)\left(1-\delta_{2}\right) \\
& \geq(1+\epsilon / 2) \log n, \quad(*)
\end{aligned}
$$


where we increase $N_{0}$, if necessarily to guarantee the validity of $(*)$. Therefore for all $n \geq N_{0}$, we obtain

$$
P\left(A_{n}\right) \leq \frac{3 n}{2 \beta} \frac{e^{-\left(1+\delta_{0}\right) \log n}}{\sqrt{\frac{4 \pi \log n}{\beta^{2}}}}<3 n^{-\epsilon / 2} .
$$

Using the increasing sequence of integers, $n_{j}:=\max \left\{n:\left[\left(2 \beta^{-2} \log n\right)^{1 / \alpha}\right]=j\right\}$.

Notice that, for $n_{j-1}<n \leq n_{j}$, we have $\left[\left(2 \beta^{-2} \log n\right)^{1 / \alpha}\right]=j$ and $D_{n}\left(r_{n}\right) \leq D_{n_{j}}\left(r_{n_{j}}\right)$. Thus,

$$
P\left(A_{n} \quad \text { i.o. in } \mathrm{n}\right) \leq P\left(A_{n_{j}} \text { i.o. in } \mathrm{j}\right) \text {. }
$$

So, for all $n \geq N_{0}$, we obtain by the integral test

$$
\sum_{j=N_{2}}^{\infty} P\left(A_{n_{j}}\right) \leq \sum_{j=N_{0}}^{\infty} 3 n_{j}^{-\epsilon / 2} \leq 3 \sum_{j=N_{0}}^{\infty} e^{-\epsilon j^{\alpha} / 2}<\infty .
$$

Therefore, Borel-Canteli lemma implies that $P\left(A_{n_{j}}(\epsilon)\right.$ i.o. in $\left.\mathrm{j}\right)=0$. Let $\epsilon \downarrow 0$ and recall the relation (??), we conclude that

$$
P\left\{\limsup _{n \rightarrow \infty}\left(\begin{array}{c}
r_{n} \\
m
\end{array}\right)^{-1} \frac{D_{n}\left(r_{n}\right)}{m \zeta_{1} r_{n}^{\frac{\alpha-1}{2}}} \leq \beta\right\}=1 .
$$

To complete the proof, we need to show that

$$
P\left\{\liminf _{n \rightarrow \infty}\left(\begin{array}{c}
r_{n} \\
m
\end{array}\right)^{-1} \frac{D_{n}\left(r_{n}\right)}{m \zeta_{1} r_{n}^{\frac{\alpha-1}{2}}} \geq \beta\right\}=1 .
$$

For that, set $\tilde{A}_{n}:=\left\{D_{n}\left(r_{n}\right) \leq(1-\epsilon) \beta\left(\begin{array}{c}r_{n} \\ m\end{array}\right) r_{n}^{\frac{\alpha-1}{2}} m \zeta_{1}\right\}$. Then

$$
\begin{aligned}
P\left(\tilde{A}_{n}\right) & =P\left\{D_{n}\left(r_{n}\right) \leq(1-\epsilon) \beta\left(\begin{array}{c}
r_{n} \\
m
\end{array}\right) r_{n}^{\frac{\alpha-1}{2}} m \zeta_{1}\right\} \\
& =P\left\{\max _{0 \leq k \leq n-r_{n}} U_{1+k}^{k+r_{n}} \leq(1-\epsilon) \beta\left(\begin{array}{c}
r_{n} \\
m
\end{array}\right) r_{n}^{\frac{\alpha-1}{2}} m \zeta_{1}\right\} \\
& \leq P\left\{\bigcap_{k=1}^{\left[\frac{n}{r_{n}}\right]} U_{1+(k-1) r_{n}}^{k r_{n}} \leq(1-\epsilon) \beta\left(\begin{array}{c}
r_{n} \\
m
\end{array}\right) r_{n}^{\frac{\alpha-1}{2}} m \zeta_{1}\right\}
\end{aligned}
$$




$$
\begin{aligned}
& =\left(P\left\{\frac{r_{n}}{m}\left(\begin{array}{c}
r_{n} \\
m
\end{array}\right)^{-1} U_{1}^{r_{n}} \leq(1-\epsilon) \beta \zeta_{1} r_{n}^{\frac{\alpha+1}{2}}\right\}\right)^{\left[\frac{n}{r_{n}}\right]} \\
& =\left(P\left(U_{r_{n}} \leq \beta(1-\epsilon) \zeta_{1} r_{n}^{\frac{\alpha+1}{2}}\right)\right)^{\left[\frac{n}{r_{n}}\right]}
\end{aligned}
$$

Using Corollary ??, we obtain

$$
\begin{aligned}
P\left(U_{r_{n}} \leq \beta(1-\epsilon) \zeta_{1} r_{n}^{\frac{\alpha+1}{2}}\right) & =1-P\left(U_{r_{n}}>\beta(1-\epsilon) \zeta_{1} r_{n}^{\frac{\alpha+1}{2}}\right) \\
& \leq 1-\frac{e^{\frac{-\beta^{2}(1-\epsilon) r_{n}^{2}}{2}\left(1+\left|\eta_{r_{n}}\right|\right)}}{2 \beta(1-\epsilon) \sqrt{2 \pi r_{n}^{\alpha}}} .
\end{aligned}
$$

Since the sequence $\eta_{r_{n}} \rightarrow 0$ and $r_{n}^{\alpha} \leq\left(\frac{2 \log n}{\beta^{2}}\right)$, we have for all $n \geq N_{3}$

$$
\begin{aligned}
& \frac{\beta^{2}}{2}(1-\epsilon)^{2} r_{n}^{\alpha}\left(1+\left|\eta_{r_{n}}\right|\right) \leq \frac{\beta^{2}}{2}(1-\epsilon)^{2}\left(\frac{2 \log n}{\beta^{2}}\right)\left(1+\left|\eta_{r_{n}}\right|\right) \\
& \leq \frac{\beta^{2}}{2}(1-\epsilon)^{2}\left(\frac{2 \log n}{\beta^{2}}\right)\left(1+\delta_{3}\right) \\
& \leq(1-3 \epsilon) \log n, \quad(* *)
\end{aligned}
$$

where we increase $N_{3}$, if necessary to guarantee $(* *)$. Therefore

$$
\begin{aligned}
P\left(U_{r_{n}} \leq \beta(1-\epsilon) \zeta_{1} r_{n}^{\frac{\alpha+1}{2}}\right) & \leq 1-(2 \beta)^{-1}\left(\frac{4 \pi \log n}{\beta^{2}}\right)^{-1 / 2} n^{-(1-3 \epsilon)} \\
& \leq 1-(16 \pi \log n)^{-1 / 2} n^{-(1-3 \epsilon)} \\
& \leq 1-n^{-(1-2 \epsilon)} \leq e^{-n^{-(1-2 \epsilon)}}
\end{aligned}
$$

where we increase $N_{3}$ if necessary, so that $(16 \pi \log n)^{-1 / 2} \geq n^{-\epsilon}$. It follows that for all $n \geq N_{3}$

$$
P\left(\tilde{A}_{n}\right) \leq e^{-\left[\frac{n}{r_{n}}\right] n^{-(1-2 \epsilon)}} \leq e^{-n^{\epsilon}},
$$

where we take $N_{3}$ large enough so that $r_{n} \leq n^{\epsilon}$ and $\left[\frac{n}{r_{n}}\right] \geq n^{1-\epsilon}$. Then we obtain

$$
\sum_{n=N_{3}}^{\infty} P\left(\tilde{A}_{n}\right) \leq \sum_{n=N_{3}}^{\infty} e^{-n^{\epsilon}}<\infty .
$$


Hence, Borel-Canteli lemma implies that

$$
P\left\{\liminf _{n \rightarrow \infty} \frac{1}{m \zeta_{1}} r_{n}^{\frac{-(\alpha-1)}{2}}\left(\begin{array}{c}
r_{n} \\
m
\end{array}\right)^{-1} D_{n}\left(r_{n}\right) \geq \beta(1-\epsilon)\right\}=1
$$

Since $\epsilon>0$ is arbitrary, consequently as $\epsilon \downarrow 0$

$$
P\left\{\liminf _{n \rightarrow \infty} \frac{1}{m \zeta_{1}} r_{n}^{\frac{-(\alpha-1)}{2}}\left(\begin{array}{c}
r_{n} \\
m
\end{array}\right)^{-1} D_{n}\left(r_{n}\right) \geq \beta\right\}=1
$$

which completes the proof of the theorem.

Note that Theorem ?? assumed the validity of the condition $E e^{a\left|h\left(X_{1}, \ldots, X_{m}\right)\right|}<\infty$ for some positive number $a$. In many cases such a condition does not holds, but under some restriction on the kernel $h$, such a condition can be relaxed. In fact we can formulate and proof the following theorem.

Consider the statistics

$$
D_{n}^{*}\left(r_{n}\right):=\max _{0 \leq k \leq n-r_{n}} \sum_{k+1 \leq i_{1}<\ldots<i_{m} \leq k+r_{n}}\left(X_{i_{1}} \cdots . X_{i_{m}}-\mu^{m}\right) .
$$

Theorem 5.1.3. (Generalized Erdös-Rényi law for nondegenerate U-Statistics with product kernels) Consider a U-statistics based on the kernel $h$ given by $h\left(x_{1}, \ldots, x_{m}\right)=\prod_{i=1}^{m} x_{i}$. Assume that $E X_{1} \neq 0$ and $E e^{t\left|X_{1}\right|}<\infty$ for some $t>0$. Let $r_{n}=\left[\left(2 \beta^{-2} \log n\right)^{1 / \alpha}\right]$, where $0<\alpha<1$. Then, for each $\beta>0$

$$
\frac{D_{n}^{*}\left(r_{n}\right)}{\left(\begin{array}{c}
r_{n} \\
m
\end{array}\right) r_{n}^{\frac{\alpha-1}{2}} m \sigma|\mu|^{m-1}} \rightarrow \beta \quad \text { a.s. }
$$

Proof: The proof is identical to the proof of Theorem ??.

Notice that, in Theorem ??, if $m=1$ and $\sigma^{2}=1$, then we get Book Theorem ??. Thus, Theorem ?? contain ?? as a special case.

\subsection{Strong laws for large increments of $U$ statistics}

In this section, we are going to describe the a.s. asymptotic behavior of the statistics introduced by (??) - (??) considering large increments, i.e., the sequence $r_{n}$ in the defini- 
tion of these statistics satisfying the relation $\frac{r_{n}}{\log n} \rightarrow \infty$. We give several lemmas towards that goal. That is, we extend Csörgö -Révéz law for U-statistics partly, which generalizes the Csörgö -Révéz law for sums of i.i.d. random variables Theorem ?? given by Frolov [?]. The proof is heavily depending on Corollary ?? for the nondegenerate U-statistics, and Proposition ?? together with the martingale property of $U_{1+k}^{k+r_{n}}$ for arbitrary U-statistics.

Lemma 5.2.1. Consider a nondegenerate U-statistics $U_{n}(h)$ with $E h\left(X_{1}, \ldots, X_{m}\right)=0$ and $0<\zeta_{1}^{2}<\infty$. Suppose that

$$
E e^{t\left|h\left(X_{1}, \ldots, X_{m}\right)\right|^{1 / \lambda}}<\infty \quad \text { for } \lambda \geq 2 \text { and } t \in\left[-t_{0}, t_{0}\right] .
$$

Furthermore consider a nondecreasing sequence of natural numbers $a_{n} \leq n$ such that $\frac{a_{n}}{(\log n)^{2 \lambda-1}} \rightarrow \infty$. Set $\hat{b}_{n}:=\sqrt{2 m^{2} \zeta_{1}^{2} a_{n}\left(\log \left(n / a_{n}\right)+\log \log n\right)}$ and $\hat{B}_{n}:=\left(\begin{array}{c}a_{n} \\ m\end{array}\right) \frac{\hat{b}_{n}}{a_{n}}$. Then

$$
\limsup _{n \rightarrow \infty} \frac{W_{n}\left(a_{n}\right)}{\hat{B}_{n}} \geq \limsup _{n \rightarrow \infty} \frac{\tilde{R}_{n}\left(a_{n}\right)}{\hat{B}_{n}} \geq \limsup _{n \rightarrow \infty} \frac{T_{n}\left(a_{n}\right)}{\hat{B}_{n}} \geq 1 \quad \text { a.s. }
$$

If in addition $(i) \lim _{n \rightarrow \infty} \frac{\log \left(\frac{n}{a_{n}}\right)}{\log \log n}=\infty$ is satisfied. Then

$$
\liminf _{n \rightarrow \infty} \frac{W_{n}\left(a_{n}\right)}{\hat{B}_{n}} \geq \liminf _{n \rightarrow \infty} \frac{D_{n}\left(a_{n}\right)}{\hat{B}_{n}} \geq 1 \quad \text { a.s. }
$$

Proof. Let $\epsilon>0$ be arbitrary and set $\beta=(1-\epsilon)$ in Corollary ??. Define $A_{n}:=\left\{U_{n+1}^{n+a_{n}} \geq(1-\epsilon) \hat{B}_{n}\right\}$, then

$$
\begin{aligned}
P\left(A_{n}(\epsilon)\right) & =P\left(a_{n}\left(\begin{array}{c}
a_{n} \\
m
\end{array}\right)^{-1} \sum_{n+1 \leq i_{1}<\ldots<i_{m} \leq n+a_{n}} h\left(X_{i_{1}}, \ldots, X_{i_{m}}\right) \geq(1-\epsilon) \hat{b}_{n}\right) \\
& =P\left(a_{n} U_{a_{n}}(h) \geq(1-\epsilon) \hat{b}_{n}\right) \\
& \geq\left(\frac{a_{n}}{n \log n}\right)^{(1+\delta)(1-\epsilon)^{2}} \operatorname{using}(? ?) \\
& \geq\left(\frac{a_{n}}{n \log n}\right)^{(1-\epsilon / 2)},
\end{aligned}
$$

for all large $n$, whenever $\delta$ is small enough. First suppose that, $a_{n} / n \rightarrow \tau \in[0,1)$. Define the nondecreasing sequence $n_{p}$ as follows:

Choose a natural number $N_{0}$ such that $\frac{a_{n}+1}{n}<\rho$, where $\rho \in(\tau, 1)$. Set $n_{1}=N_{0}$ and for $p=1,2, \ldots$ set $n_{p+1}:=\min \left\{n: n \geq n_{p}+a_{n}\right\}$. Making use of the inequality $-\log (1-x) \leq$ 
$C_{\rho} x$ for $x \in(0, \rho)$ and a constant $C_{\rho}$. Fix a natural number $k$, then it follows

$$
\begin{aligned}
\sum_{p=2}^{k} P\left(A_{n_{p}}\right) & \geq \sum_{p=2}^{k}\left(\frac{a_{n_{p}}}{n_{p} \log n_{p}}\right)^{(1-\epsilon / 2)} \\
& \geq\left(\log n_{k}\right)^{\frac{\epsilon}{2}-1} \sum_{p=2}^{k}\left(\frac{a_{n_{p}}}{n_{p}}\right) \\
& \geq\left(\log n_{k}\right)^{\frac{\epsilon}{2}-1} \sum_{p=2}^{k}\left(\frac{a_{n_{p}}+1}{2 n_{p}}\right) \\
& \geq\left(1 / 2 C_{\rho}\right)\left(\log n_{k}\right)^{\frac{\epsilon}{2}-1} \sum_{p=2}^{k}-\log \left(1-\frac{a_{n_{p}}+1}{n_{p}}\right) \\
& =\left(1 / 2 C_{\rho}\right)\left(\log n_{k}\right)^{\frac{\epsilon}{2}-1} \sum_{p=2}^{k} \log \left(\frac{n_{p}}{n_{p}-a_{n_{p}}-1}\right) \\
& \geq\left(1 / 2 C_{\rho}\right)\left(\log n_{k}\right)^{\frac{\epsilon}{2}-1} \sum_{p=2}^{k} \log \left(\frac{n_{p}}{n_{p-1}}\right) \text { since } n_{p}-a_{n_{p}}-1<n_{p-1} \\
& \geq C\left(\log n_{k}\right)^{\frac{\epsilon}{2}-1} \log \prod_{p=2}^{k} \frac{n_{p}}{n_{p-1}} \\
& \geq\left(\log n_{k}\right)^{\frac{\epsilon}{2}-1} \log n_{k} \\
& =C\left(\log n_{k}\right)^{\epsilon / 2} \rightarrow \infty, \quad a s \quad k \rightarrow \infty .
\end{aligned}
$$

Since the events $A_{n_{p}}$ are independent, the assertion of (??) in this case follows by the second Borel-Canteli lemma.

Finally suppose that, $a_{n} / n \rightarrow 1$ as $n \rightarrow \infty$. Then for $n$ large we have

$$
P\left(A_{n}\right) \geq(1 / 2)^{1-\epsilon / 2}(\log n)^{\frac{\epsilon}{2}-1} .
$$

Set $n_{p}=\left[\theta^{p}\right]$, where $\theta>3$. It follows that

$$
\begin{aligned}
\sum_{p=2}^{k} P\left(A_{n_{p}}\right) & \geq(1 / 2)^{1-\epsilon / 2} \sum_{p=2}^{k}\left(\left(\log n_{p}\right)^{\frac{\epsilon}{2}-1}\right. \\
& \geq(1 / 2)^{1-\epsilon / 2} \sum_{p=2}^{k}(p \log \theta)^{\frac{\epsilon}{2}-1} \rightarrow \infty
\end{aligned}
$$

as $k \rightarrow \infty$. Since $n_{p+1}-n_{p} \geq 2 \theta^{p}>a_{n_{k}}$, then the events $A_{n_{p}}$ are independent. An application of the second Borel-Canteli lemma completes the assertion of (??) of the 
lemma. To prove the assertion of (??), let $\epsilon>0$ be arbitrary and set $\beta=(1-\epsilon)$ in Corollary ??. Define the events

$$
\begin{aligned}
\tilde{A}_{n}:= & \left\{\max _{0 \leq k \leq n-a_{n}} U_{k+1}^{k+a_{n}} \leq(1-\epsilon) \hat{B}_{n}\right\} \text {. Then, } \\
P\left(\tilde{A}_{n}\right) & \leq P\left(\bigcap_{k=1}^{\left[n / a_{n}\right]} a_{n}\left(\begin{array}{c}
a_{n} \\
m
\end{array}\right)^{-1} U_{1+(k-1) a_{n}}^{k a_{n}} \leq(1-\epsilon) \hat{b}_{n}\right) \\
& =\left(P\left(a_{n} U_{a_{n}}(h) \leq(1-\epsilon) \hat{b}_{n}\right)\right)^{\left[\frac{n}{a_{n}}\right]} \\
& =\left(1-P\left(a_{n} U_{a_{n}}>(1-\epsilon) \hat{b}_{n}\right)\right)^{\left[\frac{n}{a_{n}}\right]} \\
& \leq\left(1-\left(\frac{a_{n}}{n \log n}\right)^{(1+\delta)(1-\epsilon)^{2}}\right)^{\left[\frac{n}{a_{n}}\right]} \\
& \leq e^{-\left[\frac{n}{a_{n}}\right]\left(\frac{a_{n}}{n \log n}\right)^{1-\epsilon / 2}} \text { using }(? ?) \\
& \leq e^{\left(-n / a_{n}\right)\left(n / a_{n}\right)^{-1+\epsilon / 2}(\log n)^{-1+\epsilon / 2}} \\
& \leq e^{-C\left(n / a_{n}\right)^{\epsilon / 2}(\log n)^{-1+\epsilon / 2}} \\
& \leq e^{-C_{1}(\log n)^{1+\epsilon / 2}}=O\left(n^{-2}\right),
\end{aligned}
$$

where we choose $\delta$ in Corollary ?? to be small enough such that $(1+\delta)(1-\epsilon)^{2} \geq 1-\epsilon / 2$. The last inequality follows by using (i). Hence Borel-Canteli lemma implies the result.

Remark 5.2.1. If we are able to prove that $\lim _{\sup _{n \rightarrow \infty}} \frac{W_{n}\left(a_{n}\right)}{\hat{B}_{n}} \leq 1$, then we get equality in (??) and (??). Observe that the equality in (??) will contradict the equality in (??) (see Lemma ?? below). However we expect that equality in (??) can be achieved but limit point in Lemma ?? may be zero.

Lemma 5.2.2. Consider a U-statistics $U_{n}(h)$ with kernel $h\left(X_{1}, \ldots, X_{m}\right)$ satisfying the condition (H2) and $0<\zeta_{m}^{2}:=E h^{2}\left(X_{1}, \ldots, X_{m}\right)<\infty$. Furthermore consider a nondecreasing sequence of natural numbers $a_{n} \leq n$ such that $\frac{a_{n}}{\log n} \rightarrow \infty$. Set $\tilde{b}_{n}:=\sqrt{2 m \zeta_{m}^{2} a_{n}\left(\log \left(n / a_{n}\right)+\log \log n\right)}$ and $\tilde{B}_{n}:=\left(\begin{array}{c}a_{n} \\ m\end{array}\right) \frac{\tilde{b}_{n}}{a_{n}}$. Then

$$
\limsup _{n \rightarrow \infty} \frac{R_{n}\left(a_{n}\right)}{\tilde{B}_{n}} \leq \limsup _{n \rightarrow \infty} \frac{D_{n}\left(a_{n}\right)}{\tilde{B}_{n}} \leq 1 \quad \text { a.s. }
$$


Proof. Let $\epsilon>0$ be arbitrary and in Proposition ?? set $\beta=(1+\epsilon)$. Define $A_{n}:=$ $\left\{\max _{0 \leq k \leq n-a_{n}} U_{k+1}^{k+a_{n}} \geq(1+\epsilon) \tilde{B}_{n}\right\}$. Then,

$$
\begin{aligned}
& P\left(A_{n}\right)=P\left(\max _{0 \leq k \leq n-a_{n}} U_{k+1}^{k+a_{n}} \geq(1+\epsilon) \tilde{B}_{n}\right) \\
& =P\left(\max _{0 \leq k \leq n-a_{n}}\left(\begin{array}{c}
a_{n} \\
m
\end{array}\right)^{-1} \sum_{k+1 \leq i_{1}<\ldots<i_{m} \leq k+a_{n}} h\left(X_{i_{1}}, \ldots, X_{i_{m}}\right) \geq(1+\epsilon) \frac{\tilde{b}_{n}}{a_{n}}\right) \\
& \leq e^{-t(1+\epsilon) \frac{\tilde{b}_{n}}{a_{n}}} E e^{t U_{a_{n}}(h)} \quad \text { since } U_{a_{n}}(h) \text { is reverse martingale }(t>0) \\
& \leq e^{-\left[\frac{n}{m}\right] \sup _{s}\left(s(1+\epsilon) \frac{\tilde{b}_{n}}{a_{n}}-\log E e^{s h\left(X_{1}, \ldots, X_{m}\right)}\right)}, \quad \text { by using same arguments in Lemma ?? } \\
& =e^{-\left[\frac{n}{m}\right] \xi_{h}\left((1+\epsilon) \tilde{b}_{n} / a_{n}\right)} \quad \text { where } \xi_{h}() \text { defined in }(? ?) \\
& \leq\left(\frac{a_{n}}{n \log n}\right)^{(1+\epsilon)^{2}(1-\delta)}, \quad \text { by using Proposition } ? ? \\
& \leq\left(\frac{a_{n}}{n \log n}\right)^{(1+\epsilon / 2)} \\
& \leq(\log n)^{-(1+\epsilon / 2)},
\end{aligned}
$$

where we choose $\delta$ in Proposition ?? to be small enough such that $(1-\delta)(1+\epsilon)^{2} \leq(1+\epsilon / 2)$ and using the fact that $a_{n} \leq n$. Let $\theta>1$ and for $p=1,2, \ldots$ set $n_{p}:=\left[\theta^{p}\right]$. Then

$$
\sum_{p=1}^{\infty} P\left(A_{n_{p}}\right)=\sum_{p=1}^{\infty}\left(\log \left[\theta^{p}\right]\right)^{-(1+\epsilon / 2)}<\infty \quad \text { for all } \epsilon>0 .
$$

Therefore by Borel-Canteli lemma, we get

$$
\limsup _{p \rightarrow \infty} \frac{D_{n_{p}}\left(a_{n_{p}}\right)}{\tilde{B}_{n_{p}}} \leq 1 \quad \text { a.s. }
$$

The properties of $a_{n}$ and the definition of $\tilde{B}_{n}$ implies that $\tilde{B}_{n_{p}} \leq \tilde{B}_{n_{p+1}} \leq \theta \tilde{B}_{n_{p}}$. Hence, whenever $n_{p} \leq n<n_{p+1}$, it follows that

$$
\frac{D_{n_{p}}\left(a_{n_{p}}\right)}{\theta \tilde{B}_{n_{p}}} \leq \frac{D_{n}\left(a_{n}\right)}{\tilde{B}_{n}} \leq \theta \frac{D_{n_{p+1}}\left(a_{n_{p+1}}\right)}{\tilde{B}_{n_{p+1}}} .
$$

Since $\theta$ may be chosen arbitrary close to 1 , the assertion of the lemma follows. 


\subsubsection{Extended Erdös-Rényi Law for U-statistics}

In this subsection, we are interested in studying the a.s. asymptotic behavior of (??)-(??) when the increments are very short in the sense that $r_{n}$ in the definition of statistics (??) - (??) satisfies the relation $r_{n} / \log n \rightarrow 0$. Thus, we will derive a result for Ustatistics, which contain Theorem ?? of Mason (1989) as a special case. For this purpose recall the notation given by (??).

Lemma 5.2.3. Assume that, the kernel $h$ satisfies the condition (H2). Then for any nondecreasing sequence $k_{n}$ such that $c_{n}:=\frac{\log n}{k_{n}} \rightarrow \infty$, we have

$$
\limsup _{n \rightarrow \infty} \frac{D_{n}\left(k_{n}\right)}{m\left(\begin{array}{c}
k_{n} \\
m
\end{array}\right) \gamma_{h}\left(c_{n}\right)} \leq 1 \quad \text { a.s. }
$$

where $\gamma_{h}\left(c_{n}\right):=\sup \left\{z: \xi_{h}(z) \leq c_{n}\right\}$.

Proof. For any integer $i \geq 1$, let $\Omega_{i}:=\left\{n: k_{n}=i\right\}$ and for any integer $j \geq 0$, let $\Gamma_{j}:=\left\{n: 2^{j} \leq n<2^{j+1}\right\}$. Furthermore, let $E_{i, j}=\Omega_{i} \cap \Gamma_{j}$ and $\beta_{j}:=\sum_{i=1}^{\infty} 1_{\left\{E_{i, j} \neq \phi\right\}}$. If $E_{i, j} \neq \phi$, let $\beta_{i, j}:=\min \left\{n \in E_{i, j}\right\}$. Notice that if $E_{i, j} \neq \phi$, then

$$
\begin{aligned}
& P\left(\max _{n \in E_{i, j}}\left(\begin{array}{c}
i \\
m
\end{array}\right)^{-1} \frac{D_{n}(i)}{m \gamma_{h}\left(\frac{\log n}{i}\right)} \geq 1+\epsilon\right)= \\
& =P\left(\max _{n \in E_{i, j}} \max _{0 \leq p \leq n-i}\left(\begin{array}{c}
i \\
m
\end{array}\right)^{-1}\left(m \gamma_{h}\left(\frac{\log n}{i}\right)\right)^{-1} \sum_{1+p \leq i_{1}<\ldots<i_{m} \leq p+i} h\left(X_{i_{1}}, \ldots, X_{i_{m}}\right) \geq 1+\epsilon\right) \\
& \leq P\left(\max _{0 \leq p \leq 2^{j+1}-i}\left(\begin{array}{c}
i \\
m
\end{array}\right)^{-1} \sum_{1+p \leq i_{1}<\ldots<i_{m} \leq p+i} h\left(X_{i_{1}}, \ldots, X_{i_{m}}\right) \geq m(1+\epsilon) \gamma_{h}\left(\frac{\log \beta_{i, j}}{i}\right)\right) \\
& \leq 2^{j+1} P\left(\left(\begin{array}{c}
i \\
m
\end{array}\right)^{-1} \sum_{1 \leq i_{1}<\ldots<i_{m} \leq i} h\left(X_{i_{1}}, \ldots, X_{i_{m}}\right) \geq m(1+\epsilon) \gamma_{h}\left(\frac{\log \beta_{i, j}}{i}\right)\right) \\
& =2^{j+1} P\left(U_{i}(h) \geq m(1+\epsilon) \gamma_{h}\left(\frac{\log \beta_{i, j}}{i}\right)\right. \\
& \leq 2^{j+1} e^{-(1+\epsilon) \log \beta_{i, j}} \quad \text { by using Proposition ?? } \\
& \leq 2^{j+1} e^{-(1+\epsilon) \log 2^{j}} \text { by definition of } \beta_{i, j}
\end{aligned}
$$

Therefore, by the above estimate we get

$$
P\left(\max _{n \in \Gamma_{j}}\left(\begin{array}{c}
k_{n} \\
m
\end{array}\right)^{-1} \frac{D_{n}\left(k_{n}\right)}{m \gamma_{h}\left(c_{n}\right)} \geq 1+\epsilon\right)=
$$




$$
\begin{aligned}
& =P\left(\max _{n \in \cup_{i=1}^{\infty} E_{i, j}}\left(\begin{array}{c}
k_{n} \\
m
\end{array}\right)^{-1} \frac{D_{n}\left(k_{n}\right)}{m \gamma_{h}\left(c_{n}\right)} \geq 1+\epsilon\right) \\
& \leq \beta_{j} 2^{j+1} e^{-(1+\epsilon) j \log 2} \\
= & 2 \beta_{j} 2^{-\epsilon j} \leq(2(j+1) \log 2) 2^{-\epsilon j}
\end{aligned}
$$

This is because $\beta_{j} \leq k_{2^{j+1}}$ and, thus, $c_{n} \rightarrow \infty$ implies $\beta_{j} \leq(j+1) \log 2$ for all sufficiently large $j$. Hence, Borel-Cantelli lemma completes the proof.

Recall the definition of the sequences $a_{n}, a(x), \beta_{n}$ and $b_{n}$. Set $B_{n}:=\left(\begin{array}{c}a_{n} \\ m\end{array}\right) \Lambda\left(\frac{\beta_{n}}{a_{n}}\right)$. Based on this notation, we are going to prove the following lemma, which describe the upper limit of (??) - (??) for all sequences $a_{n}$ so that $\frac{\log a_{n}}{\log n} \rightarrow 0$.

Lemma 5.2.4. Let the kernel $h$ satisfying condition (H2). Assume that there exists a function $g(\theta), \theta \in\left(1, \theta_{0}\right)$, such that $g(\theta) \rightarrow 1$ as $\theta \rightarrow 1$ and $\frac{B(x \theta)}{B(x)} \leq g(\theta)$ for all sufficiently large $x$. Then

$$
\limsup _{n \rightarrow \infty} \frac{R_{n}\left(a_{n}\right)}{m B_{n}} \leq \limsup _{n \rightarrow \infty} \frac{D_{n}\left(a_{n}\right)}{m B_{n}} \leq \limsup _{n \rightarrow \infty} \frac{W_{n}\left(a_{n}\right)}{m B_{n}} \leq 1 \quad \text { a.s. }
$$

Proof: Write $\beta_{n}:=\log \left(n / a_{n}\right)+\log \log n$. Then

$$
\begin{aligned}
& P\left\{W_{n}\left(a_{n}\right) \geq m(1+\epsilon) B_{n}\right\}= \\
& =P\left\{\max _{0 \leq j \leq n-a_{n}} \max _{m \leq k \leq a_{n}} \sum_{1+j \leq i_{1}<\ldots<i_{m} \leq k+j} h\left(X_{i_{1}}, \ldots, X_{i_{m}}\right) \geq m(1+\epsilon)\left(\begin{array}{c}
a_{n} \\
m
\end{array}\right) \gamma_{h}\left(\frac{\beta_{n}}{a_{n}}\right)\right\} \\
& \leq n P\left\{\max _{m \leq k \leq a_{n}} \sum_{1 \leq i_{1}<\ldots<i_{m} \leq k} h\left(X_{i_{1}}, \ldots, X_{i_{m}}\right)>\left(\begin{array}{c}
a_{n} \\
m
\end{array}\right) m(1+\epsilon) \gamma_{h}\left(\frac{\beta_{n}}{a_{n}}\right)\right\} \\
& \leq n \sum_{k=m}^{a_{n}} P\left\{\sum_{1 \leq i_{1}<\ldots<i_{m} \leq k} h\left(X_{i_{1}}, \ldots, X_{i_{m}}\right) \geq\left(\begin{array}{c}
k \\
m
\end{array}\right) m(1+\epsilon) \gamma_{h}\left(\frac{\beta_{n}}{k}\right)\right\} \\
& \leq n \sum_{k=m}^{a_{n}} e^{-(1+\epsilon) \beta_{n}} \quad \operatorname{using} \operatorname{Proposition} ? ? \\
& =n a_{n}\left(\frac{n}{a_{n}}\right)^{-(1+\epsilon)}(\log n)^{-(1+\epsilon)} \\
& =n^{-\epsilon} a_{n}^{2+\epsilon}(\log n)^{-(1+\epsilon)} \leq(\log n)^{-(1+\delta)} \quad(*) \quad \text { for some } \delta>0 .
\end{aligned}
$$


Making use of the sequence $n_{k}:=\left[\theta^{k}\right]$, it follows from $(*)$ and Borel-Canteli lemma that,

$$
\limsup _{k \rightarrow \infty} \frac{W_{n_{k}}\left(a_{n_{k}}\right)}{m B_{n_{k}}} \leq 1 \quad \text { a.s. }
$$

Further since $B_{n_{k}} \leq B_{n_{k+1}} \leq B_{n_{k}} g(\theta)$ for all sufficiently large $k$, also because $W_{n}\left(a_{n}\right)$ is nondecreasing, it follows that

$$
\limsup _{n \rightarrow \infty} \frac{W_{n}\left(a_{n}\right)}{m B_{n}} \leq g(\theta) \text { a.s. }
$$

Now if we take the limit when $\theta \rightarrow 1$ and observe that $R_{n}\left(a_{n}\right) \leq D_{n}\left(a_{n}\right) \leq W_{n}\left(a_{n}\right)$, we get the desired assertion.

Notice that, if we can prove that $\lim _{\sup _{n \rightarrow \infty}} \frac{T_{n}\left(a_{n}\right)}{m B_{n}} \geq 1 \quad$ a.s., then a.s. the asymptotic behavior of (??) - (??) for all such $a_{n}$. 


\section{Chapter 6}

\section{Summary and Open Questions}

This thesis presented a brief historical overview of the strong law for increments of sums of i.i.d. random variables including the famous two laws, the Erdös-Rényi law and the Csörgö-Révéz law. We developed these laws to more general situation by considering increments of U-statistics and obtained the Erdös-Rényi law as well as the Csörgö-Révéz law for U-statistics. We introduced two different types of statistics based on increments of U-statistics in (??), (??) and we defined also other functionals of these types. Taking $h(x, y)=(x+y) / 2$ and $h(x)=x$ we got the statistics defined by Erdös and Rényi, Shepp, and Csörgö and Révéz as a special case of our more general Statistics defined in (??) and (??) respectively.

- In Section ?? we described the a.s. asymptotic behavior of the statistic $\hat{D}_{n}\left(a_{n}\right)$ and other functionals of this type (defined in (??) and (??) - (??)) considering increments of length $a_{n}$. In fact, $a_{n}$ has to grow at most as $n$, and at least as $(\log \log n)^{2}$ or $\sqrt{(\log n)^{1+\delta}}$ depending on the distribution function of $h_{1}\left(X_{1}\right)$. We did that only for nondegenerate U-statistics.

Open questions to be further investigated:

(1) Determine the a.s. asymptotic behavior of the statistic $\hat{D}_{n}\left(r_{n}\right)$, where $m \leq$ $r_{n}<\sqrt{(\log n)^{1+\delta}}$ for all $n$ and $\delta>0$ for nondegenerate U-statistics. This is very important from extreme value theory point of view.

(2) Describe the a.s. asymptotic behavior of the statistic $\hat{D}_{n}\left(r_{n}\right)$, when the Ustatistics under consideration are degenerate, and $r_{n}$ be any nondecreasing sequence of natural numbers such that $m \leq r_{n} \leq n$ for all $n$.

(3) Find the rate of convergence of the statistic $\hat{D}_{n}\left(r_{n}\right)$. To this problem, we may use our methods as given in Section ?? together with the result given by Deheuvels et. al. [?], if $r_{n}=[c \log n]$ for some positive number $c$ and if $r_{n}$ chosen such that $\frac{r_{n}}{(\log n)^{2}}$, then using the results of Csörgö and Steinebach [?] to answer such problem, provided the U-statistics are nondegenerate. 
- in Section ?? Lemma ?? we proved that

$$
\limsup _{n \rightarrow \infty} \frac{1}{n} \log P\left(U_{n}(h) \geq x\right) \leq \frac{-1}{m} \Delta(x),
$$

and used it to established the upper limit of Erdös-Rényi law as well as CsörgöRévéz law for arbitrary U-statistics of degree $m$. It is very important in further investigation to prove the following statement

$$
\liminf _{n \rightarrow \infty} \frac{1}{n} \log P\left(U_{n}(h) \geq x\right) \geq \frac{-C}{m} \Delta(x),
$$

where $C$ is positive constant. Such a statement will enable us to give a direct proof, if we want to study the a.s. asymptotic behavior of the statistic $D_{n}\left(r_{n}\right)$ and the other functionals of this type defined in (??) and (??) - (??) respectively, (see the proof of Theorem ??).

Remarkable results in this direction are given by Nikitin and Ponikarov [?, ?] where their theorem cover bounded kernel (degenerate and nondegenerate) for both oneand two samples U-statistics. Also some results in that direction given by Eichelsbacher and Löwe [?] or Eichelsbacher [?] or Shuya Kanagawa [?]. All of these result may be used in describing the a.s. asymptotic behavior of the statistic $D_{n}\left(r_{n}\right)$ and the other functionals of this type as well as to give the rate of convergence.

In subsection ?? Lemma ?? we extended Mason Theorem ?? partly, that means we proved only the upper limit for the statistic $D_{n}\left(r_{n}\right)$ with $\frac{r_{n}}{\log n} \rightarrow 0$. From the viewpoint of extreme value theory for $m$ dependent random variables it is of interest to investigate the lower limit. 


\section{Bibliography}

[1] Ahmed, I.A. (1981) On some asymptotic properties of U-statistics. Scand. J. Statist. Theory and Appl. 8 No.3, 175-181.

[2] Aleskeviciene, A. (1990) Probabilities of large deviations for U-statistics and the functional of Mises. Theory Probab. Appl. 35(1).

[3] Aleskeviciene, A. (1992). Large deviations for U-statistics. Lithuanian Math. J. 32(1).

[4] Arcones, M. A. (1992) Large deviation for U-statistics. J. Multivariate Anal. 42(2) 299-301.

[5] Arcones, M. A. (1995) A Bernstein-type inequality for U-statistics and U-processes. Statistics and Probability Letters 22 223-230.

[6] Arcones, M. A., Giné, E. (1993). Limit theorems for U-processes. Annals of Probability 21 1494-1542.

[7] Arratia, R., and Waterman, M. S., (1989). Erdös-Rényi strong law for pattern matching with a given proportion of mismatches. Annals of Probability 17(3) 1152-1169.

[8] Bahadur, R. R. and R. Range Rao (1960) On deviations of the sample mean. Ann. Math. Statist. 31 1015-1027.

[9] Bauer, H. (1990). Wahrscheinlichkeitstheorie. Springer-Verlag, Berlin.

[10] Baringhaus, L. and Rank, R. (2003). On large deviations of U-statistics and their projections. Sankhyá, Series A. 64 167-170.

[11] Berk, R. H. (1966) Limiting behavior of posterior distributions when the model is incorrect. Ann. Math. Statist. 37, No 1, 51-58.

[12] Binswanger, K. (1994) Longest runs in coin tossing. Insurance: Math and Economics. 15 139-149. 
[13] Book, S.A. (1975). An extension of the Erdös-Rényi law of large numbers. Proc. Amer. Math. Soc. 48 438-446.

[14] Borovskikh, Yu. V. (1996). U-statistics in Banach spaces. VSP, Utrecht.

[15] Borovskikh, Yu. V. and Weber, N. C. (2003). Large deviations of U-statistics. I. Lithuanian Mathematical Journal. 43 11-33.

[16] Borovskikh, Yu. V. and Weber, N. C. (2003). Large deviation results for a Ustatistical sum with product kernel. Bulletin Austral. Math. Soc. (to appear).

[17] Charalambides, Ch. A., Koutras, M.V., Balakrishnan, N. (2001). Probability and statistical models with applications. Chapman and Hall/CRC. 101-113.

[18] Chernoff, H. (1965) A measure of asymptotic efficiency for tests of hypothesis based on the sum of observations. Ann. Math. Statist. 23 493-507.

[19] Christofides, T.C. (1991) Probability inequalities with exponential bounds for Ustatistics. Statist. Probab. Lett. 12 257-261.

[20] Chow, Y. S. and Teicher, H. (1988). Probability Theory: Independent, Interchangeability, Martingales; Second edition. Springer-Verlag, New York.

[21] Csörgö, M. and Révéz, P. (1979) How big are the increments of a Wiener process. Ann. Probab. 7 731-737.

[22] Csörgö, M. and Révéz, P. (1981) Strong approximation in probability and Statistics, Akadémiai Kiadó, Budapest; Academic Press, New York.

[23] Csörgö, S. (1979) Erdös-Rényi laws.

[24] Csörgö, M. and Steinebach, J. (1981) Improved Erdös-Rényi and strong approximation laws for increments of partial sums. Ann. Probab. 9(6) 988-996. Ann. Statist. 7 772-787.

[25] Deheuvels, P., Devroye, L. and Lynch, J. (1986) Exact Convergence rate in the limit theorems of Erdös-Rényi and Shepp. Ann. Probab. 14 209-223.

[26] Deheuvels, P. and Devroye, L. (1987) limit laws of Erdös-Rényi-Shepp type. Ann. Probab. 15 1363-1386.

[27] Denker, M. (1985). Distribution Theory in Nonparametric Statistics. Vieweg, Braunschweig.

[28] Deuschel, J.-D. and Stroock, D. W. (1989) Large Deviations, Academic Press, London. 
[29] Eichelsbacher, P. and Löwe, M. (1995). A large deviation principle for m-variate von Mises-statistics and U-statistics. J. Theoret. Probab. 8 807-824.

[30] Eichelsbacher, P. (2005). Refined large deviations for von-Mises statistics. Theory of Probability and its Application. 49(1) 139-148.

[31] Erdös, P. and Rényi, A. (1970). On a new law of large numbers. J. Analyse Math. 23 103-111.

[32] Frolov, A.N. (1998) One-sided strong laws for large increments of sums. Statist. Probab. Lett. 37 155-165.

[33] Frolov, A.N. (2002) One-sided strong laws for increments of sums of i.i.d. random variables. Studia Sci. Math. Hungar. 39 333-395.

[34] Frolov, A.N. (2003) Limit theorems for increments of sums of independent random variables. Theory Probab. Appl. 48 93-107

[35] Hoeffding, W. (1961) The strong law of large numbers for U-statistics. Inst. Statist. Mimeo Ser., No. 302 1-10.

[36] Halmos, P.R. (1946) The theory of unbiased estimation. Ann. Math. Statist. 17 34-43.

[37] Hoeffding, W. (1963) Probability inequalities for sum of bounded random variables. J. Amer. Statist. Assoc. 58 13-30.

[38] Hollander, F. den. (2000). Large Deviations. Fields Institute Monographs, Toronto.

[39] Keener, R. V., Robinson, J. and Weber, C. N. (1998) Tail probability approximation for U-statistics. Statist. Probab. Lett. 37 59-65.

[40] Korolyuk, V.S. and Borovskikh, Yu.V. (1994). Theory of U-Statistics.

[41] Lee, A.J. (1990). U-Statistics. Marcel Deker Inc. New York.

[42] Löwe, M. (1992). Exponential inequalities and principle of large deviation for Ustatistics and von Mises-statistics, Dissertation. Universität Bielefeld.

[43] Lynch, J. (1983). Some comments of the Erdös-Rényi law and a theorem of Shepp. Ann. Probab. 11 801-802.

[44] Kanagawa, S. (1999). A representation of the rate functions in large deviations principles for U-statistics with degenerate kernels. Trends in Probability and Related Analysis. 229-239. 
[45] Malevich, T. L. and Abdalimov, B. (1979). Probabilities of large deviations for Ustatistics. Theory Probab. Appl. 24 215-220.

[46] Mason, D.M. (1989). An extended version of the Erdös-Rényi strong law of large numbers. Ann. Probab. 17 257-265.

[47] Nikitin, Ya. Yu. and Ponikarov, E.V. (1999) Rough asymptotic of probabilities of Chernoff type large deviations for von Mises functionals and U-statistics. Proceedings of St. Petersburg Mathematical Society 7 124-167.

[48] Nikitin, Ya. Yu. and Ponikarov, E.V. (2002) Large deviations of nondegenerate twosample von Mises functionals.

[49] Petrov, V.V. (1965). On probabilities of large deviations for sum of independent random variables. Theory Probab. Appl. 10 287-298.

[50] Petrov, V.V. (1975). Sums of Independent Random Variables. Springer-Verlag, Berlin.

[51] Petrov, V.V. (1995). Limit Theorem of Probability Theory. Springer-Verlag, Berlin.

[52] Sanchis, G. R. (1994). A functional limit theorem for Erdös and Rényi law of large numbers. Probab. Theory and Relat. Fields. 98 1-5.

[53] Sen, P.K. (1981). Sequential Nonparametrics: Invariance Principles and Statistical Inference. Wiley, New York.

[54] Serfling, R.J. (1980). Approximation Theorems of Mathematical Statistics. Wiley, New York.

[55] Shiryaev, A. N. (1996). Probability. Springer-Verlag.

[56] Shepp, L. (1965) A limit law concerning moving averages. Ann. Math. Statist. 35 424-428.

[57] Shao, Qi-Man. (1995) On a conjecture Revesz. Proc. Amer. Math. Soc. 123 575-582.

[58] Steinebach, J. (1978) On a necessary condition for the Erdös-Rényi law of large numbers. Proc. Amer. Math. Soc. 68 97-100.

[59] Steinebach, J. (1979) Erdös-Rényi Zuwächse bei Erneuerungsprozessen und Partialsummen auf Gitterin. Habilitationsschrift, University Düsseldorf. 68 97-100.

[60] Steinebach, J. (1981). Large Deviation Probabilities And Some Related Topics. Carleton Mathematical Lecture Notes No. 28. 
[61] Steinebach, J. (1986) Improved Erdös-Rényi and strong approximation laws for increments of renewal processes. Proc. Ann. Probab. 14 (No. 2) 547-559.

[62] Stout, W.F. (1974). Almost Sure Convergence. Academic, New York.

[63] Vandemaele, M. (1982). On probabilities of large deviations for U-statistics. Theory Probab. Appl. 27614.

[64] Vandemaele, M. and Veraverbeke (1982). Cramer type large deviations for linear cominations of order statistics. Ann. Probab. 10 423-434.

[65] Vandemaele, M. and Veraverbeke, D. (1985). Cramer type large deviations for studentized U-statistics. Metrica. 32 165-180.

[66] von Mises, R. (1947) On the asymptotic distribution of differentiable statistical functions. Ann. Math. Statist. 18 309-348. 